УДК 515.12

\author{
I. HLushak, O. NyKYFORCHYN
}

\title{
FRACTAL DIMENSIONS FOR INCLUSION HYPERSPACES AND NON-ADDITIVE MEASURES
}

\begin{abstract}
I. Hlushak, O. Nykyforchyn. Fractal dimensions for inclusion hyperspaces and non-additive measures, Mat. Stud. 50 (2018), 3-21.

Analogues of Hausdorff dimension, upper and lower box dimensions for the inclusion hyperspaces and non-additive regular measures (capacities) on metric compacta are introduced. Their relations to the respective dimensions of sets and additive measures are investigated. Methods for finding and estimating fractal dimensions of self-similar inclusion hyperspaces and self-similar non-additive measures are presented.
\end{abstract}

1. Introduction. Dimension functions are the cornerstone of fractal theory and an important tool of topological dynamics. A wide variety of dimensions were introduced not only for sets in metric and topological spaces, but also for set functions, mostly for Borel measures in metric spaces $[3,8]$. There is relative agreement on which properties of dimensions for measures are mandatory and which are desirable [6]. Among the most widely used are Hausdorff dimension, box dimension, packing dimension, correlation dimension et al.

Usually a dimension of a regular additive measure describes "how dimensional" can be a part of the underlying space which carries "most of" the measure. Unfortunately, this approach is completely inappropriate for non-additive measures, or capacities, which were introduced by Choquet [2] as a natural generalization of measures. They found numerous applications, e.g. in decision making theory in conditions of uncertainty [10]. Upper semicontinuous capacities were defined and studied in [5]. Algebraic and topological properties of capacities on compact Hausdorff spaced were investigated in [12].

The key drawback with the non-additive measures is that the same set can be "light" in one circumstance and "heavy" in another. It is easy to construct a capacity $c$ on a compactum $X$ and disjoint closed sets $A, B$, and $C$ such that the difference $c(A \cup C)-c(A)$ is small and the difference $c(B \cup C)-c(B)$ is large. To overcome this obstacle, we define a notion of a foundation for an (additive or non-additive) measure, in particular, for an inclusion hyperspace [13], which can be equivalently regarded as a non-additive measure that attain only values 0 and 1 . This results in analogues of Hausdorff dimension, upper box dimension, and lower box dimension. We are going to apply the developed tool to self-similar inclusion hyperspaces and self-similar non-additive measures, which were introduced in [7].

2. Basic definitions, notation and facts. A compactum is a compact Hausdorff topological space. In the sequel we restrict our attention to metric compacta. Although capacities

2010 Mathematics Subject Classification: 28A78, 28A80, 54F45.

Keywords: Hausdorff dimension; box dimension; measure; capacity; self-similarity; iterated function system. doi:10.15330/ms.50.1.3-21

(C) I. Hlushak, O. Nykyforchyn, 2018 
can also be considered on Tychonoff spaces, which include all metric spaces, difficulties in this more general case arise so fast that this can overshadow our main ideas. Therefore we defer noncompact case to future publications.

We regard the unit segment $I=[0 ; 1]$ as a subspace of the real line with the natural topology. We write $A \underset{\mathrm{cl}}{\subset} B$ or $A \underset{\mathrm{op}}{\subset} B$ if $A$ is a closed or resp. open subset of a space $B$.

For a topological space $X$ its hyperspace $\exp X$ is the set of all closed nonempty subsets of $X$ with the Vietoris topology, see, e.g., [13]. The standard base of the latter consists of all sets of the form

$$
\left\langle U_{1}, \ldots, U_{n}\right\rangle=\left\{F \in \exp X \mid F \subset U_{1} \cup \cdots \cup U_{n}, F \cap U_{i} \neq \varnothing \text { for all } i=1, \ldots, n\right\},
$$

where $U_{1}, \ldots, U_{n}$ are open sets in $X$. If $(X, d)$ is a metric compactum, the Vietoris topology on $\exp X$ is determined by the Hausdorff metric $d_{H}$ that is defined as

$$
d_{H}(F, G)=\inf \{\varepsilon \geqslant 0 \mid d(a, B) \leqslant \varepsilon, d(b, A) \leqslant \varepsilon \text { for all } a \in A, b \in B\}, \quad F, G \in \exp X,
$$

where $d(x, Y)=\inf \{d(x, y) \mid y \in Y\}$ for any $x \in X, Y \in \exp X$. It is known that for a compactum $X$ the hyperspace $\exp X$ is a compactum as well, therefore we can consider the compacta $\exp ^{2} X=\exp (\exp X), \exp ^{3} X=\exp \left(\exp ^{2} X\right)$, etc. For a metric compactum $(X, d)$ the Vietoris topology on $\exp ^{2} X$ is determined by the metric $d_{H H}=\left(d_{H}\right)_{H}$, and so forth.

For $\delta \geqslant 0$ and a set $A$ in a metric space $(X, d)$ let $\bar{O}_{\delta}(A)=\{x \in X \mid d(x, A) \leqslant \delta\}$. Then we can equivalently define the Hausdorff metric by the formula

$$
d_{H}(F, G)=\min \left\{\delta \geqslant 0 \mid F \subset \bar{O}_{\delta}(G), G \subset \bar{O}_{\delta}(F)\right\} .
$$

The diameter of a set $A$ in a metric space $(X, d)$ is defined to be $\operatorname{diam} A=\sup \{d(x, y) \mid$ $x, y \in A\}$.

An inclusion hyperspace $\mathcal{G}$ on a compactum $X$ is a non-empty closed subset of $\exp X$ such that $A \in \mathcal{H}, A \subset B$ imply $B \in \mathcal{G}$ for all $A, B \in \exp X$ (see [13]). The set $G X$ of all inclusion hyperspaces on $X$ is closed in $\exp ^{2} X$, therefore $G X$ is a compactum. If $(X, d)$ is a metric compactum, then the topology of $G X$ is determined by the restriction of the metric $d_{H H}$, which for inclusion hyperspaces $\mathcal{G}, \mathcal{G}^{\prime}$ satisfies

$$
d_{H H}\left(\mathcal{G}, \mathcal{G}^{\prime}\right)=\inf \left\{\varepsilon \geqslant 0 \mid \bar{O}_{\varepsilon} A \in \mathcal{G}^{\prime} \text { for all } A \in \mathcal{G}, \bar{O}_{\varepsilon} B \in \mathcal{G} \text { for all } B \in \mathcal{G}^{\prime}\right\}
$$

Each element $A$ of an inclusion hyperspace $\mathcal{G}$ contains a minimal element $A_{0} \in \mathcal{G}$, i.e., an element such that $A_{0} \supset B \in \mathcal{G}$ implies $A_{0}=B$. An inclusion hyperspace consists of the closed supersets of its minimal elements.

For an inclusion hyperspace $\mathcal{G} \in G X$, among all $X_{0} \underset{\text { cl }}{\subset} X$ such that $A \in \mathcal{G}$ implies $A \cap X_{0} \in \mathcal{G}$, there is a smallest set, which is called the support of $\mathcal{G}$ and denoted by supp $\mathcal{G}$.

It is well known [13] that, for an inclusion hyperspace $\mathcal{G}$, its transversal $\mathcal{G}^{\perp}=\{T \in$ $\exp X \mid T \cap A \neq \varnothing$ for all $A \in \mathcal{G}\}$ is an inclusion hyperspace as well, and the transversal mapping $(-)^{\perp}: G X \rightarrow G X$ that takes each $\mathcal{G}$ to $\mathcal{G}^{\perp}$ is an involutive homeomorphism, which preserves the supports.

We follow a terminology of [12] and call a function $c: \exp X \cup\{\varnothing\} \rightarrow I$ a capacity $^{1}$ (or a regular non-additive measure) on a compactum $X$ if the following properties hold for all closed subsets $F, G$ in $X$ :

Not to be confused with the so-called Carathéodory capacities [8]. 
1. $c(\varnothing)=0$;

2. if $F \subset G$, then $c(F) \leqslant c(G)$ (monotonicity); and

3. if $c(F)<a$, then there exists an open set $U \supset F$ such that for any $G \subset U$ we have $c(G)<a$ (upper semicontinuity).

A capacity $c$ on $X$ is called normalized if $c(X)=1$, and subnormalized if $c(X) \leqslant 1$.

We extend a capacity $c$ to all open subsets in $X$ by the formula:

$$
c(U)=\sup \{c(F) \mid F \underset{\mathrm{cl}}{\subset} X, F \subset U\} .
$$

It is proved in [12] that the set $M X$ of all normalized capacities on a compactum $X$ is a compactum as well, if a topology on $M X$ is determined with a subbase that consists of all sets of the form

$$
O_{-}(F, a)=\{c \in M X \mid c(F)<a\}
$$

where $F \underset{\text { cl }}{\subset} X, a \in \mathbb{R}$, and

$O_{+}(U, a)=\{c \in M X \mid c(U)>a\}=\{c \in M X \mid$ there exists a compactum $F \subset U, c(F)>a\}$, where $U \underset{\text { op }}{\subset} X, a \in \mathbb{R}$. Similarly the set $\underline{M} X$ of all subnormalized capacities can be turned into a compactum: the respective subbase is of the form $\underline{O}_{-}(F, a)=\{c \in \underline{M} X \mid c(F)<a\}$, where $F \underset{\mathrm{cl}}{\subset} X, a \in \mathbb{R}$, and

$\underline{O}_{+}(U, a)=\{c \in \underline{M} X \mid c(U)>a\}=\{c \in \underline{M} X \mid$ there exists a compactum $F \subset U, c(F)>a\}$, for $U \subset X, a \in \mathbb{R}$.

If the topology on a compactum $X$ is determined with a compatible metric $d$, then [12] the topology on $M X$ (or on $\underline{M X}$ ) is determined with the following metric:

$$
\hat{d}\left(c, c^{\prime}\right)=\inf \left\{\varepsilon>0 \mid \forall F \underset{\mathrm{cl}}{\subset} X c\left(\bar{O}_{\varepsilon}(F)\right)+\varepsilon \geqslant c^{\prime}(F), c^{\prime}\left(\bar{O}_{\varepsilon}(F)\right)+\varepsilon \geqslant c(F)\right\} .
$$

For a capacity $c \in \underline{M} X$, among all such $X_{0} \underset{\mathrm{cl}}{\subset} X$ that $c(A)=c\left(A \cap X_{0}\right)$ for all $A \underset{\mathrm{cl}}{\subset} X$, there is a smallest set, which is called the support of $c$ and denoted by $\operatorname{supp} c$.

If $c$ is a normalized capacity on $X$, then the set function

$$
\tilde{c}(A)=1-\sup \{c(B) \mid B \underset{\mathrm{cl}}{\subset} X, A \cap B=\varnothing\}, \quad A \underset{\mathrm{cl}}{\subset} X,
$$

is a normalized capacity on $X$ as well. It is called the conjugate (or dual) capacity to $c$, and the conjucacy mapping $\varkappa X: M X \rightarrow M X$ that takes each $c$ to $\tilde{c}$ is an involutive autohomeomorphism, which preserves the supports.

Remark 1. Observe that, being restricted to the subsets $P X$ of probability measures and $\underline{P} X$ of subprobability measures, the considered topologies and metrics on $M X$ and $\underline{M} X$ induce respectively the well known weak* topology and the Prokhorov metric.

Remark 2. The space $G X$ can be considered as a subspace of $M X$ that consists of the normalized capacities that attain only the values 0 and 1 . Namely, each $\mathcal{G} \in G X$ determines the capacity

$$
c_{\mathcal{G}}(F)=\left\{\begin{array}{l}
1, F \in \mathcal{G}, \\
0, F \notin \mathcal{G},
\end{array} \quad \text { for all } F \underset{\mathrm{cl}}{\subset} X,\right.
$$

and $\hat{d}\left(c_{\mathcal{G}}, c_{\mathcal{G}^{\prime}}\right)=\min \left\{d_{H H}\left(\mathcal{G}, \mathcal{G}^{\prime}\right), 1\right\}$ for all inclusion hyperspaces $\mathcal{G}, \mathcal{G}^{\prime}$. 
It is important that the constructions $G$ and $M$ are functors: for a continuous mapping $f: X \rightarrow Y$ of compacta the continuous mappings $G f: G X \rightarrow G Y$ and $M f: M X \rightarrow M Y$ are defined as follows:

$$
\begin{gathered}
G f(\mathcal{G})=\{B \underset{\mathrm{cl}}{\subset} Y \mid B \supset f(A) \text { for some } A \in \mathcal{G}\}, \mathcal{G} \in G X, \\
M f(c)(B)=c\left(f^{-1}(B)\right), \quad c \in M X, B \underset{\mathrm{cl}}{\subset} Y .
\end{gathered}
$$

3. Dimensions for inclusion hyperspaces. We start with the inclusion hyperspaces, which are the simplest (bi-valued) non-additive measures. Although an inclusion hyperspace is a set of sets, it is useless to consider its topological dimension. For example, an inclusion hyperspace in $I$ can contain a copy of the Hilbert cube, hence is infinite-dimensional w.r.t. all sensible points of view. Therefore we wish to know how many small sets are necessary to approximate the elements of an inclusion hyperspace. From now on $X$ is a metric compactum.

Definition 1. For an inclusion hyperspace $\mathcal{G} \in G X$, a finite or countable collection $\mathcal{F}$ of closed sets in $X$ is called a foundation of $\mathcal{G}$ if, whenever $A \in \mathcal{G}$, there are $F_{1}, F_{2}, \ldots, F_{n} \in \mathcal{F}$, $n \in \mathbb{N}$, such that $F_{i} \cap A \neq \varnothing$, for all $1 \leqslant i \leqslant n$, and $F_{1} \cup F_{2} \cup \cdots \cup F_{n} \in \mathcal{G}$.

Definition 2. A foundation $\mathcal{F}$ of $\mathcal{G}$ is called a $\delta$-foundation, for $\delta>0$, if $\operatorname{diam} F \leqslant \delta$ for all $F \in \mathcal{F}$.

Having a $\delta$-foundation $\mathcal{F}$ for an inclusion hyperspace $\mathcal{G}$ and a set $A \underset{\text { cl }}{\subset} X$, we verify if there are $F_{1}, F_{2}, \ldots, F_{n} \in \mathcal{F}, n \in \mathbb{N}$, such that $F_{i} \cap A \neq \varnothing$, for all $1 \leqslant i \leqslant n$, and $F_{1} \cup F_{2} \cup \cdots \cup F_{n} \in \mathcal{G}$.

If there are no such $F_{i} \in \mathcal{F}$, then $A$ is not in $\mathcal{G}$.

If such $F_{i} \in \mathcal{F}$ exist, then we cannot be sure that $A \in \mathcal{G}$, but $A^{\prime}=A \cup F_{1} \cup F_{2} \cup \cdots \cup F_{n} \in \mathcal{G}$. Observe that $A \subset A^{\prime} \subset \bar{O}_{\delta} A$, hence $d_{H}\left(A, A^{\prime}\right) \leqslant \delta$.

Thus we have determined an inclusion hyperspace $\mathcal{G}$ on $X$ approximately "in a finite way". It is sufficient to specify only which finite unions of elements of a $\delta$-foundation $\mathcal{F}$ belong to $\mathcal{G}$. Using this information, we can decide whether an arbitrary set $A \underset{\text { cl }}{\subset} X$ is in $\mathcal{G}$, or at least we can know if there exist a set $A^{\prime} \in \mathcal{G}$ that is sufficiently close to $A$ w.r.t. Hausdorff distance. For finite $\mathcal{F}$ the inclusion hyperspace $\mathcal{G}$ is approximately determined with a list of $<2^{|\mathcal{F}|}$ subsets of $X$.

Remark 3. For $\mathcal{F}$, to be a foundation of $\mathcal{G}$, it is sufficient, but not necessary, that there are $F_{1}, F_{2}, \ldots, F_{n} \in \mathcal{F}$ such that $F_{1} \cup F_{2} \cup \cdots \cup F_{n} \supset \operatorname{supp} \mathcal{G}$. On the other hand, if $\mathcal{F}$ is a foundation of $\mathcal{G}$, then $\{F \in \mathcal{F} \mid F \cap \operatorname{supp} \mathcal{G} \neq \varnothing\}$ is a foundation of $\mathcal{G}$ as well.

For $\mathcal{G} \in G X, s \geqslant 0, \delta>0$, we denote:

$$
\begin{gathered}
N_{\delta}(\mathcal{G})=\min \{|\mathcal{F}| \mid \mathcal{F} \text { is a finite } \delta \text {-foundation of } \mathcal{G}\}, \\
\mathcal{H}_{\delta}^{s}(\mathcal{G})=\inf \left\{\sum\left\{(\operatorname{diam} F)^{s} \mid F \in \mathcal{F}\right\} \mid \mathcal{F} \text { is a } \delta \text {-foundation of } \mathcal{G}\right\} .
\end{gathered}
$$

Now, as it is usually done, we put $\mathcal{H}^{s}(\mathcal{G})=\lim _{\delta \rightarrow 0} \mathcal{H}_{\delta}^{s}(\mathcal{G})$, and define the upper box dimension, the lower box dimension, and the Hausdorff dimension of $\mathcal{G}$ as follows:

$$
\overline{\operatorname{dim}}_{B} \mathcal{G}=\varlimsup_{\delta \rightarrow 0} \frac{\ln N_{\delta}(\mathcal{G})}{-\ln \delta}, \quad \underline{\operatorname{dim}}_{B} \mathcal{G}=\varliminf_{\delta \rightarrow 0} \frac{\ln N_{\delta}(\mathcal{G})}{-\ln \delta}
$$




$$
\operatorname{dim}_{H} \mathcal{G}=\sup \left\{s \geqslant 0 \mid \mathcal{H}^{s}(\mathcal{G})=\infty\right\}=\inf \left\{s \geqslant 0 \mid \mathcal{H}^{s}(\mathcal{G})=0\right\}
$$

Obviously $\operatorname{dim}_{H} \mathcal{G} \leqslant \underline{\operatorname{dim}}_{B} \mathcal{G} \leqslant \overline{\operatorname{dim}}_{B} \mathcal{G}$ for all $\mathcal{G} \in G X$. If the values of $\underline{\operatorname{dim}}_{B} \mathcal{G}$ and $\overline{\operatorname{dim}}_{B} \mathcal{G}$ coincide, then we call them the box dimension of $\mathcal{G}$ and denote $\operatorname{dim}_{B} \mathcal{G}$. Remark 3 implies also the inequalities $\operatorname{dim}_{H} \mathcal{G} \leqslant \operatorname{dim}_{H} \operatorname{supp} \mathcal{G}, \underline{\operatorname{dim}}_{B} \mathcal{G} \leqslant \underline{\operatorname{dim}}_{B} \operatorname{supp} \mathcal{G}, \overline{\operatorname{dim}}_{B} \mathcal{G} \leqslant$ $\overline{\operatorname{dim}}_{B} \operatorname{supp} \mathcal{G}$.

Thus the introduced fractal dimensions describe asymptotic behaviors of the minimal cardinality and of the minimal "smallness" (in terms of sum of powers of diameters) of $\delta$ foundations for an inclusion hyperspace when $\delta$ tends to 0 .

Remark 4. It is easy to give an example of a countable $\delta$-foundation $\mathcal{F}=\left\{F_{1}, F_{2}, F_{3}, \ldots\right\}$ of an inclusion hyperspace $\mathcal{G}$ such that none of its finite subfamilies is a $\delta$-foundation. On the other hand, for any sequence $\varepsilon_{n} \searrow 0$ the family $\mathcal{F}^{\prime}=\left\{\bar{O}_{\varepsilon_{1}} F_{1}, \bar{O}_{\varepsilon_{2}} F_{2}, \bar{O}_{\varepsilon_{3}} F_{3}, \ldots\right\}$ is a $\delta+2 \varepsilon_{1}$-foundation from which we can choose a finite subfamily that is a $\delta+2 \varepsilon_{1}$-foundation as well. This implies that in the definition of $\mathcal{H}_{\delta}^{s}(\mathcal{G})$ and therefore in the definition of the Hausdorff dimension of $\mathcal{G}$ we can consider finite $\delta$-foundations only.

Proposition 1. If $\mathcal{G} \in G X$ has a unique minimal element $A_{0} \underset{\mathrm{cl}}{\subset} X$, i.e., $A_{0} \in \mathcal{G}$ and $A \underset{\mathrm{cl}}{\subset} X$, $A \in \mathcal{G}$ implies $A \supset A_{0}$, then $\operatorname{dim}_{H} \mathcal{G}=\operatorname{dim}_{H} A_{0}, \underline{\operatorname{dim}}_{B} \mathcal{G}=\underline{\operatorname{dim}}_{B} A_{0}, \overline{\operatorname{dim}}_{B} \mathcal{G}=\overline{\operatorname{dim}}_{B} A_{0}$.

Proof. A collection $\mathcal{F}$ of closed sets is a foundation of $\mathcal{G}$ if and only if it contains a finite subcover of $A_{0}$.

Proposition 2. There is an inclusion hyperspace $\mathcal{G}$ in $I^{2}$ such that $\underline{\operatorname{dim}}_{B} \mathcal{G}=\overline{\operatorname{dim}}_{B} \mathcal{G}<$ $\underline{\operatorname{dim}}_{B} \operatorname{supp} \mathcal{G}=\overline{\operatorname{dim}}_{B} \operatorname{supp} \mathcal{G}$.

Proof. Let, for $n \in \mathbb{N}, x_{n}=\left(\frac{1}{n}, 0\right), y_{n}=\left(\frac{1}{n}, \frac{1}{2^{n}}\right)$, and $X=\left\{x_{n} \mid n \in \mathbb{N}\right\} \cup\left\{y_{n} \mid n \in\right.$ $\mathbb{N}\} \cup\{(0,0)\}$.

We construct a hyperspace $\mathcal{G}$ with the support $X$ as follows: $A \underset{\text { cl }}{\subset} I^{2}$ is in $\mathcal{G}$ if and only if it contains either of the sets: $A_{0}=\left\{x_{k} \mid k \in \mathbb{N}\right\} \cup\{(0,0)\}, A_{n}=\left\{x_{1}, x_{2}, \ldots, x_{n}, y_{n}\right\}$ for $n \in \mathbb{N}$. It follows from Example 3.5 [3] that $\underline{\operatorname{dim}}_{B} X=\overline{\operatorname{dim}}_{B} X=\underline{\operatorname{dim}}_{B}\left\{0,1, \frac{1}{2}, \frac{1}{3}, \ldots\right\}=$ $\overline{\operatorname{dim}}_{B}\left\{0,1, \frac{1}{2}, \frac{1}{3}, \ldots\right\}=\frac{1}{2}$.

Let $\delta>0, n \in \mathbb{N}$ be such that $\frac{1}{2^{n}} \leqslant \delta<\frac{1}{2^{n-1}}$, then it is easy to see that the set of the closed balls of the radii $\frac{1}{2^{n}}$ with the centers $x_{1}, y_{1}, x_{2}, y_{2}, \ldots, x_{n-1}, y_{n-1}, x_{n}$ is a $\delta$-foundation of $\mathcal{G}$. Hence $N_{\delta}(\mathcal{G}) \leqslant 2 n-1 \leqslant 2\left[-\log _{2} \delta\right]+1$, and

$$
\overline{\operatorname{dim}}_{B} \mathcal{G}=\varlimsup_{\delta \rightarrow 0} \frac{\ln N_{\delta}(\mathcal{G})}{-\ln \delta} \leqslant \varlimsup_{\delta \rightarrow 0} \frac{\ln \left(2\left[-\log _{2} \delta\right]+1\right)}{-\ln \delta} \leqslant \varlimsup_{t \rightarrow+\infty} \frac{\ln (2 t+1)}{t \ln 2}=0,
$$

which implies $\underline{\operatorname{dim}}_{B} \mathcal{G}=\overline{\operatorname{dim}}_{B} \mathcal{G}=0$.

Question 1. Is there an inclusion hyperspace $\mathcal{G}$ such that the strict inequality $\operatorname{dim}_{H} \mathcal{G}<$ $\operatorname{dim}_{H} \operatorname{supp} \mathcal{G}$ is valid? Note that, if for each $\delta>0$ a $\delta$-foundation of $\mathcal{G}$ is fixed, then $\varliminf_{d \rightarrow 0}\left(\bigcup \mathcal{F}_{\delta}\right) \supset \operatorname{supp} \mathcal{G}$.

Proposition 3. Let $\mathcal{F}$ be a foundation of $\mathcal{G} \in G X$ and $\mathcal{F}^{\prime}$ a collection of closed sets in $X$ such that each $F \in \mathcal{F}$ is contained in $\operatorname{Int} F^{\prime}$ for some $F^{\prime} \in \mathcal{F}^{\prime}$. Then $\mathcal{F}^{\prime}$ is a foundation of $\mathcal{G}^{\perp}$. 
Proof. Let $T \in \mathcal{G}^{\perp}$. Then $T$ has non-empty intersection with each $A \in \mathcal{G}$. By definition, there are $F_{1}, F_{2}, \ldots, F_{n} \in \mathcal{F}, n \in \mathbb{N}$, such that $F_{i} \cap A \neq \varnothing$, for all $1 \leqslant i \leqslant n$, and $F_{1} \cup F_{2} \cup \cdots \cup F_{n} \in \mathcal{G}$, hence $T$ has non-empty intersection with $F_{1} \cup F_{2} \cup \cdots \cup F_{n}$ as well. Denote by $F_{A}$ an element $F_{i}$ such that $T \cap F_{i} \neq \varnothing$, and by $F_{A}^{\prime}$ an element of $\mathcal{F}^{\prime}$ such that $F_{A} \subset \operatorname{Int} F_{A}^{\prime}$. Then the compactum $\mathcal{G} \underset{\text { cl }}{\subset} \exp X$ is covered with the open sets $\left\langle X\right.$, $\left.\operatorname{Int} F_{A}^{\prime}\right\rangle$, for $A \in \mathcal{G}$, hence there is a finite subcover $\left\langle X, \operatorname{Int} F_{A_{1}}^{\prime}\right\rangle, \ldots,\left\langle X, \operatorname{Int} F_{A_{k}}^{\prime}\right\rangle$. Then $T \cap F_{A_{j}}^{\prime} \neq \varnothing$ for $1 \leqslant j \leqslant k$, and $F_{A_{1}}^{\prime} \cup F_{A_{2}}^{\prime} \cup \cdots \cup F_{A_{k}}^{\prime} \in \mathcal{G}^{\perp}$.

Corollary 1. The transversal mapping $(-)^{\perp}: G X \rightarrow G X$ preserves the lower box dimension, the upper box dimension and the Hausdorff dimension of the inclusion hyperspaces.

4. Self-similar inclusion hyperspaces. Now we consider a case when the introduced dimensions for inclusion hyperspaces coincide with the dimensions of their supports.

A mapping $S: \mathbb{R}^{n} \rightarrow \mathbb{R}^{n}$ is called a contracting similarity if there is $0<c<1$ such that $d(S(x), S(y))=c \cdot d(x, y)$ for all $x, y \in \mathbb{R}^{n}$ ( $d$ is the Euclidean distance). The number $s$ is called the ratio of $S$. Obviously such $S$ is an affine bijection. For a finite set $\mathcal{S}=\left\{S_{1}, S_{2}, \ldots, S_{k}\right\}$ of contracting similarities $\mathbb{R}^{n} \rightarrow \mathbb{R}^{n}$ the formula $A \mapsto \Phi_{\mathcal{S}}(A)=S_{1}(A) \cup S_{2}(A) \cup \cdots \cup S_{k}(A)$ defines a contracting map $\Phi_{\mathcal{S}}$ in the space $\exp \mathbb{R}^{n}$ of non-empty compact (=bounded closed) subsets of $\mathbb{R}^{n}$ with Hausdorff metric. It is well known that there is a unique fixed point for $\Phi_{\mathcal{S}}$, i.e., a non-empty compact set $K$ such that $K=S_{1}(K) \cup S_{2}(K) \cup \cdots \cup S_{k}(K)$. It is called the attractor of the iterated function system (IFS) $\mathcal{S}$.

The family $\mathcal{S}=\left\{S_{1}, S_{2}, \ldots, S_{k}\right\}$ of contracting similarities is said to satisfy Open Set Condition (OSC) if there is a non-empty bounded open set $V$ such that $S_{1}(V) \cup S_{2}(V) \cup$ $\cdots \cup S_{k}(V) \subset V$ and the above union is disjoint. If, additionally, $V$ meets the attractor $K$, then the Strong Open Set Condition (SOSC) holds. Schief [9] proved that for a finite family of contracting similarities in $\mathbb{R}^{n}$ OSC implies SOSC (it is not the case even for countable families). Then it is known [3] that $K \subset \mathrm{Cl} V$ and $\operatorname{dim}_{H} K=\underline{\operatorname{dim}}_{B} K=\overline{\operatorname{dim}}_{B} K=s$, where $s$ is the solution of the equation $c_{1}^{s}+c_{2}^{s}+\cdots+c_{k}^{s}=1, c_{i}$ are the ratios of $S_{i}$.

Similarly iterated function system for inclusion hyperspaces was defined in [7]. For a mapping $f: X \rightarrow X, X$ being a compact metric space, the contraction factor is defined to be equal to

$$
\operatorname{Lip} f=\sup \left\{\frac{d(f(x), d(y))}{d(x, y)} \mid x, y \in X, x \neq y\right\} .
$$

A mapping $f$ such that $\operatorname{Lip} f<1$ is called a contraction, and $f$ is non-expanding if $\operatorname{Lip} f \leqslant 1$. For $0<q<1$ we denote $R_{q}(X)=\{r: X \rightarrow X \mid \operatorname{Lip} r \leqslant q\}$. It is easy to see that $R_{q}(X)$ with the uniform convergence metric is a compactum.

For any $\bar{r} \in \exp R_{q}(X)$ and $\mathcal{G} \in G X$ we put $G \bar{r}(\mathcal{G})=\bigcap_{r \in \bar{r}} G r(\mathcal{G})$. Then $G \bar{r}(\mathcal{G})$ is in $G X$ and depends continuously on $(\bar{r}, \mathcal{G}) \in \exp R_{q}(X) \times G X$. Now for $\mathcal{R} \in G R_{q}(X)$ we define $G \mathcal{R}(\mathcal{F})$ by the formula $G \mathcal{R}(\mathcal{F})=\bigcup_{\bar{r} \in \mathcal{R}} G \bar{r}(\mathcal{F})$. It is easy to observe that for $H \in \exp X$ we have $H \in G \mathcal{R}(\mathcal{F})$ if and only if there is $\bar{r} \underset{\text { cl }}{\subset} R_{q}(X), \bar{r} \in \mathcal{R}$ such that for each $r \in \bar{r}$ the set $H$ contains the image $r(F)$ of some $F \in \mathcal{F}$. It is straightforward to check that $G \mathcal{R}(\mathcal{F}) \in G X$ and it depends continuously on $(\mathcal{F}, \mathcal{R}) \in G X \times M R_{q}(X)$. The defined transformation differs from the usual IFS for compact sets in that each contraction has a "choice" on which set to act in a given inclusion hyperspace. Following the commonly used terminology style (see, e.g. [11]) we call $\mathcal{R}$ an IFS for inclusion hyperspaces and $G \mathcal{R}$ an IFS operator or fractal transform associated with $\mathcal{R}$. The functors $\exp$ and $G$ preserve contraction factors of mappings, thus 
if $\mathcal{R} \in G R_{q}(X)$, then $G \mathcal{R} \in R_{q}(G X)$ [7, Theorem 1]. Hence there is a unique fixed point for $G \mathcal{R}$.

We simplify the definition to consider finite families of contracting similarities only. The parameter for the mapping $\Phi_{\mathcal{S}}$ is a finite set $\mathcal{S}$ of contracting similarities. Let $D$ be a closed subset in $\mathbb{R}^{n}$. An IFS for inclusion hyperspaces in $D$ will depend on a family $\mathbf{S}=\left\{\mathcal{S}_{1}, \mathcal{S}_{2}, \ldots, \mathcal{S}_{m}\right\}$ where each $\mathcal{S}_{i}$ is a finite set of contracting similarities $\mathbb{R}_{n} \rightarrow \mathbb{R}_{n}$ that map $D$ into itself (in fact $\mathbf{S}$ is a finite set of the minimal elements of the inclusion hyperspace $\mathcal{R}$ above). Denote $\mathcal{S}=\mathcal{S}_{1} \cup \mathcal{S}_{2} \cup \cdots \cup \mathcal{S}_{m}=\left\{S_{1}, S_{2}, \ldots, S_{k}\right\}$. Then $\mathcal{J}_{i}=\{j \in\{1,2, \ldots, k\} \mid$ $\left.\left.S_{j} \in \mathcal{S}_{i}\right\}\right\}$ is a subset of $\mathcal{J}=\{1,2, \ldots, k\}$ for all $1 \leqslant i \leqslant m$. For each $\mathcal{G} \in G D$ let the inclusion hyperspace $\Phi_{\mathbf{S}}(\mathcal{G})$ consist of all $A \underset{\text { cl }}{\subset} D$ such that there are $\mathcal{S}_{i}=\left\{S_{j_{1}}, S_{j_{2}}, \ldots, S_{j_{l}}\right\}$ and $A_{1}, A_{2}, \ldots, A_{l} \in \mathcal{G}, A \supset S_{j_{1}}\left(A_{1}\right) \cup S_{j_{2}}\left(A_{2}\right) \cup \cdots \cup S_{j_{l}}\left(A_{l}\right)$. In other words, there is $\mathcal{S}_{i}$ such that for all $S \in \mathcal{S}_{i}$ the preimage of $A$ under $S: D \rightarrow D$ is in $\mathcal{G}$. This definition is similar to the one of superfractal given in [1]. The key difference is that there is no choice of IFSs $\mathcal{S}_{1}, \mathcal{S}_{2}, \ldots, \mathcal{S}_{m}$ according to a given probability distribution, but all of them act in parallel. Thus a self-similar family of sets is obtained.

Results on $G \mathcal{R}$ [7] imply that $\Phi_{\mathbf{S}}: G D \rightarrow G D$ is a contraction, hence there is a unique fixed point $\mathcal{K}$ (the attractor of IFS $\mathbf{S}$ ). In the sequel $D$ will be the closure of a set $V$ satisfying OSC, and all inclusion hyperspaces will be regarded as families of subsets of $\mathrm{Cl} V$.

Theorem 1. Let $V \subset \mathbb{R}^{n}$ be a non-empty bounded open subset, $\mathbf{S}=\left\{\mathcal{S}_{1}, \mathcal{S}_{2}, \ldots, \mathcal{S}_{m}\right\}$ a finite family of finite sets of contracting similarities $\mathbb{R}^{n} \rightarrow \mathbb{R}^{n}$ such that:

(i) none of $\mathcal{S}_{i}$ is contained in another $\mathcal{S}_{j}$, i.e., each element of $\mathbf{S}$ is minimal w.r.t. inclusion;

(ii) Open Set Condition holds for $\mathcal{S}=\bigcup \mathbf{S}$ and $V$; and

(iii) for each element $A$ of the attractor $\mathcal{K}$ for $\mathbf{S}$ such that $A \subset \partial V$ and all $S, S^{\prime} \in \mathcal{S}, S \neq S^{\prime}$, we have $S^{\prime}(A) \not \subset S(\partial V)$.

Then for the fixed point $\mathcal{K}$ of IFS $\Phi_{\mathbf{S}}$ for inclusion hyperspaces the equality

$$
\underline{\operatorname{dim}}_{B} \mathcal{K}=\overline{\operatorname{dim}}_{B} \mathcal{K}=\operatorname{dim}_{H} \operatorname{supp} \mathcal{K}=\underline{\operatorname{dim}}_{B} \operatorname{supp} \mathcal{K}=\overline{\operatorname{dim}}_{B} \operatorname{supp} \mathcal{K}=s
$$

is valid, with $s$ being the solution of the equation $c_{1}^{s}+c_{2}^{s}+\cdots+c_{k}^{s}=1, c_{i}$ are the ratios of $S_{i} \in \mathcal{S}$.

Remark 5. Obviously, if no element of the attractor $\mathcal{K}$ for $\mathbf{S}$ is contained in the boundary of $V$, then (iii) holds. The following easier to verify property provides (iii):

(iii+) for all $S, S^{\prime} \in \mathcal{S}$ and $\mathcal{S}_{i} \in \mathbf{S}$ there is $S_{j} \in \mathcal{S}_{i}$ such that $S(\partial V) \cap S^{\prime} \circ S_{j}(\partial V)=\varnothing$.

Similarly, if

(iii++) for all $S \in \mathcal{S}$ and $\mathcal{S}_{i} \in \mathbf{S}$ there is $S_{j} \in \mathcal{S}_{i}$ such that $\partial V \cap S \circ S_{j}(\partial V)=\varnothing$

holds, then the elements of $\mathcal{K}$ are not contained in $\partial V$.

To prove the theorem, we need to introduce the following notions and notation. The $k$-tree is a non-empty set $T$ of finite sequences with elements in $\mathcal{J}=\{1,2, \ldots, k\}$. We denote such sequences $\left(j_{1}: j_{2}: \cdots: j_{r}\right), 0 \leqslant r<\infty$, hence the empty sequence () is also considered. If $\left(j_{1}: j_{2}: \cdots: j_{r}: j_{r+1}\right) \in T$, then $\left(j_{1}: j_{2}: \cdots: j_{r}\right) \in T$, and the first sequence is a child of the latter one, therefore $T$ is a $k$-ary rooted tree with the root (), $r$ is the depth of a vertex $\left(j_{1}: j_{2}: \cdots: j_{r}\right)$. Denote

$$
\left(j_{1}: j_{2}: \cdots: j_{r}: \cdot\right)=\left\{j \in \mathcal{J} \mid\left(j_{1}: j_{2}: \cdots: j_{r}: j\right) \in T\right\} .
$$


A $k$-tree $T$ is called an $\mathcal{S}$-tree if for all $\left(j_{1}: j_{2}: \cdots: j_{r}\right) \in T$ the set $\left(j_{1}: j_{2}: \cdots: j_{r}: \cdot\right)$ of indices is either empty or equal to $\mathcal{J}$, i.e., each vertex either is a leaf or has all $k$ possible children. An $\mathbf{S}$-tree is a $k$-tree $T$ such that for all $\left(j_{1}: j_{2}: \cdots: j_{r}\right) \in T$ the set $\left(j_{1}: j_{2}: \cdots\right.$ : $\left.j_{r}: \cdot\right)$ is either empty or equal to some of $\mathcal{J}_{i}$ for $1 \leqslant i \leqslant m$, i.e., each node is either a leaf or has children that correspond to all contractions in some $\mathcal{S}_{i}$. A branch of $k$-tree $T$ rooted at a vertex $\mathbf{j}=\left(j_{1}: j_{2}: \cdots: j_{r}\right) \in T$ is the $k$-tree

$$
T_{\mathbf{j}}=\left\{\left(j_{r+1}: \cdots: j_{r+q}\right) \mid\left(j_{1}: j_{2}: \cdots: j_{r}: j_{r+1}: \cdots: j_{r+q}\right) \in T, 0 \leqslant q<\infty\right\} .
$$

Obviously a branch of a $\mathcal{S}$-tree or of a $\mathbf{S}$-tree is a $\mathcal{S}$-tree or a $\mathbf{S}$-tree as well.

To attach a $k$-tree $T^{\prime}$ to a leaf $\left(j_{1}: j_{2}: \cdots: j_{r}\right)$ of a $k$-tree $T$ is to add to $T$ all vertices of the form $\left(j_{1}: j_{2}: \cdots: j_{r}: j_{1}^{\prime}: j_{2}^{\prime}: \cdots: j_{q}^{\prime}\right)$ for $\left(j_{1}^{\prime}: j_{2}^{\prime}: \cdots: j_{q}^{\prime}\right) \in T^{\prime}$.

For a set $V$ and all sequences $\left(j_{1}: j_{2}: \cdots: j_{r}\right)$ put $V_{\left(j_{1}: j_{2}: \cdots: j_{r}\right)}=S_{j_{1}}\left(V_{\left(j_{2}: j_{3}: \cdots: j_{r}\right)}\right)$, assuming $V_{()}=V$. If $\mathcal{S}=\bigcup \mathbf{S}$ and $V$ satisfy OSC, then for two sequences $\mathbf{j}$ and $\mathbf{j}^{\prime}$ we have $V_{\mathbf{j}} \subset V_{\mathbf{j}^{\prime}}$ iff $\mathbf{j}^{\prime}$ is a prefix of $\mathbf{j}, V_{\mathbf{j}^{\prime}} \subset V_{\mathbf{j}}$ iff $\mathbf{j}$ is a prefix of $\mathbf{j}^{\prime}$, otherwise $V_{\mathbf{j}}$ and $V_{\mathbf{j}^{\prime}}$ are disjoint.

Given a finite $k$-tree $T$ and an inclusion hyperspace $\mathcal{G}$, we define by induction a closed set $F_{\mathbf{j}}$ and an inclusion hyperspace $\mathcal{G}_{\mathbf{j}}$ for each vertex $\mathbf{j}=\left(j_{1}: j_{2}: \cdots: j_{r}\right) \in T$. If $\mathbf{j}$ is a leaf, then put $F_{\mathbf{j}}=\mathrm{Cl} V$ and $\mathcal{G}_{\mathbf{j}}=\mathcal{G}$, otherwise

$$
\begin{gathered}
F_{\mathbf{j}}=\bigcup\left\{S_{j}\left(F_{\left(j_{1}: j_{2}: \cdots: j_{r}: j\right)}\right) \mid j \in\left(j_{1}: j_{2}: \cdots: j_{r}: \cdot\right)\right\}, \\
\mathcal{G}_{\mathbf{j}}=\bigcap\left\{G S_{j}\left(\mathcal{G}_{\left(j_{1}: j_{2}: \cdots: j_{r}: j\right)}\right) \mid j \in\left(j_{1}: j_{2}: \cdots: j_{r}: \cdot\right)\right\} .
\end{gathered}
$$

The latter formula means that $A \in \mathcal{G}_{\mathbf{j}}$ iff there are $A_{j} \in \mathcal{G}_{\left(j_{1}: j_{2}: \cdots: j_{r}: j\right)}$ for all $j \in \mathbf{j}=\left(j_{1}\right.$ : $\left.j_{2}: \cdots: j_{r}: \cdot\right)$ such that $A \supset \bigcup_{j} S_{j}\left(A_{j}\right)$.

We denote $F_{T}=F_{()}, \mathcal{G}_{T}=\mathcal{G}_{()}$. Observe that $F_{\mathbf{j}}=F_{T_{\mathbf{j}}}, \mathcal{G}_{\mathbf{j}}=\mathcal{G}_{T_{\mathbf{j}}}$, and $F_{T}=\bigcup_{\mathbf{j} \in T} \bar{V}_{\mathbf{j}}$.

This implies that, if all minimal elements of $\mathcal{G}$ are contained in $\mathrm{Cl} V$, then all minimal elements of $\mathcal{G}_{T}$ are contained in $F_{T}$ for any $k$-tree $T$. If $T$ is a subtree of $T^{\prime}$, then $F_{T} \supset F_{T^{\prime}}$.

All families $\mathcal{S}_{i} \in \mathbf{S}, 1 \leqslant i \leqslant m$, determine $\mathbf{S}$-trees $T_{i}$ of height 1 consisting of () and all (j) for $j \in \mathcal{J}_{i}$.

In particular, for a finite $\mathbf{S}$-tree $T$ and the attractor $\mathcal{K}$ of the IFS $\mathbf{S}$ satisfying OSC for an open set $V$, the inclusion $\mathcal{K}_{T} \subset \mathcal{K}$ is valid, and for finite $\mathbf{S}$-trees $T \subset T^{\prime}$ we obtain $\mathcal{K}_{T} \supset \mathcal{K}_{T^{\prime}}$. Thus, for an infinite $\mathbf{S}$-tree, we can define

$$
F_{T}=\bigcap\left\{F_{T_{0}} \mid T_{0} \subset T \text { is a finite } \mathbf{S} \text {-tree }\right\}, \quad \mathcal{K}_{T}=\bigcap\left\{\mathcal{K}_{T_{0}} \mid T_{0} \subset T \text { is a finite } \mathbf{S} \text {-tree }\right\} .
$$

Obviously $F_{T} \in \mathcal{K}_{T}$ for an infinite $T$ as well.

On the other hand:

Lemma 1. Each minimal element $A \in \mathcal{K}$ is equal to $F_{T}$ for a unique maximal infinite $\mathbf{S}$-tree $T$ (i.e., an $\mathbf{S}$-tree $T$ without leaves, which therefore is not a subtree of any other $\mathbf{S}$-tree).

Proof. It is easy to see that $A=\bigcup_{j \in \mathcal{J}_{i}} S_{j}\left(A^{(j)}\right)$ for some $\mathcal{S}_{i}$, all $A^{(j)} \in \mathcal{K}$ are minimal. This $\mathcal{S}_{i}$ consists precisely of those $S_{j}$ that $A$ meets $V_{j}$. We represent this with the tree $T_{i}$ with the root () labeled with $A$ and each leaf $(j)$ labeled with the respective minimal set $A^{(j)}$. Each $A^{(j)}$ in turn is of the form $A^{(j)}=\bigcup_{j^{\prime} \in \mathcal{J}_{i^{\prime}}} S_{j^{\prime}}\left(A^{\left(j: j^{\prime}\right)}\right)$ for a unique $\mathcal{S}_{i^{\prime}} \in \mathbf{S}$ (namely $\left.\mathcal{S}_{i^{\prime}}=\left\{S_{j} \mid A \cap V_{\left(j: j^{\prime}\right)} \neq \varnothing\right\}\right)$ and minimal $A^{\left(j: j^{\prime}\right)} \in \mathcal{K}$, so we attach the tree $T_{j^{\prime}}$ to the leaf $(j)$ and label its leaves with the sets $A^{\left(j: j^{\prime}\right)}$. Such a process can be iterated infinitely, hence 
there is a unique maximal infinite $\mathbf{S}$-tree $T$ with all vertices $\mathbf{j}$ labeled with minimal elements $A^{\mathbf{j}}$ of $\mathcal{K}$, such that $A \in \mathcal{K}_{T_{0}}$ for any finite $\mathbf{S}$-tree $T_{0}$ that is a subtree of $T$.

Fix one of such subtrees $T_{0}$ and denote $\bar{r}$ the minimal depth of its leaves. Observe that all $A^{\mathbf{j}}$ are contained in $\bar{V}$, hence

$$
A \subset \bigcup\left\{\bar{V}_{\mathbf{j}} \mid \mathbf{j} \text { is a leaf of } T_{0}\right\}=F_{T_{0}},
$$

moreover, $A$ meets all these $\bar{V}_{\mathbf{j}}$. By definition of similarity

$$
\operatorname{diam} \bar{V}_{\left(j_{1}: j_{2}: \cdots: j_{r}\right)}=c_{j_{1}} c_{j_{2}} \ldots c_{j_{r}} \operatorname{diam} V \leqslant\left(c_{\max }\right)^{r} \operatorname{diam} V
$$

where $c_{\max }=\max \left\{c_{1}, c_{2}, \ldots, c_{k}\right\}<1$, therefore $\operatorname{diam} \bar{V}_{\mathbf{j}} \leqslant\left(c_{\max }\right)^{\bar{r}} \operatorname{diam} V$ for all leaves $\mathbf{j}$ of $T_{0}$. This implies $d_{H}\left(A, F_{T_{0}}\right) \leqslant\left(c_{\max }\right)^{\bar{r}}$ diam $V$. Taking finite subtrees $T_{0} \subset T$ with increasing minimal depth of leaves, we make $F_{T_{0}}$ converge to $F_{T}$, hence $A=F_{T}$.

Remark 6. In fact each element of the form $F_{T}$ for a maximal infinite $\mathbf{S}$-tree $T$ is minimal in $\mathcal{K}$.

In the sequel $h(A, B)$ is the one-sided Hausdorff distance between sets $A$ and $B$, i.e., sup inf $d(a, b)$. Consider a contracting similarity $S_{j} \in \mathcal{S}_{i}$ (hence $j \in \mathcal{J}_{i}$ ). Denote $a \in A \quad b \in B$

$$
\varepsilon_{i j}=\min \left\{\inf \left\{h\left(A, \partial V \cup \underset{j^{\prime} \in \mathcal{J}_{i}, j^{\prime} \neq j}{\bigcup} \bar{V}_{\left(j^{\prime}\right)}\right) \mid A \in \mathcal{K}_{T_{i^{\prime}}}\right\} \mid 1 \leqslant i^{\prime} \leqslant m, j \notin \mathcal{J}_{i^{\prime}}\right\} .
$$

Obviously it is sufficient in the above expression to consider the minimal elements $A$ of each $\mathcal{K}_{T_{i^{\prime}}}$ only. Informally speaking, $\varepsilon_{i j}$ is the measure of how much we should inflate the set $\bigcup_{j^{\prime} \in \mathcal{J}_{i}, j^{\prime} \neq j} \bar{V}_{\left(j^{\prime}\right)}=F_{T_{i} \backslash\{(j)\}}$ together with the boundary of $V$ to contain at least one of the elements of the form $\bigcup_{j^{\prime} \in \mathcal{J}_{i^{\prime}}} S_{j^{\prime}}\left(A^{\left(j^{\prime}\right)}\right), j \notin \mathcal{J}_{i^{\prime}}, A^{\left(j^{\prime}\right)} \in \mathcal{K}$, i.e., a set obtained from elements of $\mathcal{K}$ with other family $\mathcal{S}_{i^{\prime}}$ of contracting similarities not containing $S_{j}$. All $\mathcal{K}_{T_{i^{\prime}}}$ are compact, one-sided Hausdorff distance is continuous w.r.t. the "two-sided" one, hence condition (iii) ensures $\varepsilon_{i j}>0$.

Lemma 2. Let $\mathbf{j}=\left(j_{1}: j_{2}: \cdots: j_{r}\right)$ be a leaf of a finite $\mathbf{S}$-tree $T_{0}$. Then

$$
\begin{gathered}
\inf \left\{h\left(A, F_{T_{0} \backslash\{\mathbf{j}\}}\right) \mid A \in \mathcal{K}_{T}, T \text { is a finite } \mathbf{S} \text {-tree not having the vertex } \mathbf{j}\right\} \geqslant \\
\geqslant \min \left\{c_{j_{1}} c_{j_{2}} \ldots c_{j_{q}} \varepsilon_{i j_{q+1}} \mid \mathcal{J}_{i}=\left(j_{1}: j_{2}: \cdots: j_{q}: \cdot\right), 0 \leqslant q \leqslant r-1\right\} .
\end{gathered}
$$

Proof. We assume again that $A$ in the latter formula is minimal in $\mathcal{K}_{T}$. Let $q \in\{0, \ldots, r-1\}$ be maximal such that $\left(j_{1}: j_{2}: \cdots: j_{q}\right) \in T$. We represent $A$ with the tree $T$ with vertices $\mathbf{j}$ labeled with $A^{\mathbf{j}}$, as in the proof of the previous lemma. Similarly $F_{T_{0} \backslash\{\mathbf{j}\}}$ is represented with the tree $T_{0} \backslash\{\mathbf{j}\}$ with all leaves labeled with $\bar{V}$ and labels for other vertices calculated iteratively, as in the definition of $F_{\ldots . .}$.

The path

$$
() \rightarrow\left(j_{1}\right) \rightarrow\left(j_{1}: j_{2}\right) \rightarrow \cdots \rightarrow\left(j_{1}: j_{2}: \cdots: j_{q}\right)
$$

is common for the both trees $T_{0} \backslash\{\mathbf{j}\}$ and $T$. We replace all "side branches" of $T$ rooted at all the vertices of this path but the last one with the branches of the labeled tree $T_{0} \backslash\{\mathbf{j}\}$, obtaining a labeled tree $T^{\prime}$, and recalculate the labels $A^{\prime\left(j_{1}: j_{2}: \cdots: j_{q-1}\right)}, \ldots, A^{\prime\left(j_{1}\right)}, A^{\prime}=A^{\prime()}$ along the chosen path. It is easy to see that $T^{\prime}$ is "more similar" to $T_{0} \backslash\{\mathbf{j}\}$ than $T$, hence

$$
h\left(A, F_{T_{0} \backslash\{\mathbf{j}\}}\right) \geqslant h\left(A^{\prime}, F_{T_{0} \backslash\{\mathbf{j}\}}\right) .
$$


Consider the expressions

$$
F_{T_{0} \backslash\{\mathbf{j}\}}=\bigcup\left\{\bar{V}_{\mathbf{j}^{\prime}} \mid \mathbf{j}^{\prime} \text { is a leaf of } T_{0} \backslash\{\mathbf{j}\}\right\}, \quad A^{\prime}=\bigcup\left\{A_{\mathbf{j}^{\prime}}^{\prime \mathbf{j}^{\prime}} \mid \mathbf{j}^{\prime} \text { is a leaf of } T^{\prime}\right\} .
$$

Recall that, for $\mathbf{j}^{\prime}=\left(j_{1}^{\prime}: j_{2}^{\prime}: \cdots: j_{r}^{\prime}\right) \in T^{\prime}, A_{\mathbf{j}^{\prime}}^{\mathbf{j}^{\prime}}=S_{j_{1}^{\prime}} \circ S_{j_{2}^{\prime}} \circ \cdots \circ S_{j_{r}^{\prime}}\left(A^{\mathbf{j}^{\prime}}\right)$, and for a leaf $\mathbf{j}^{\prime}$ we have $A^{\mathbf{j}^{\prime}}=A^{\mathbf{j}^{\prime}}$ if $\mathbf{j}^{\prime}$ is a descendant of $\left(j_{1}: j_{2}: \cdots: j_{q}\right)$ and $A^{\mathbf{j}^{\prime}}=\bar{V}$ otherwise. Therefore the above unions differ respectively in "sub-unions"

$$
\begin{gathered}
\bigcup\left\{\bar{V}_{\mathbf{j}^{\prime}} \mid \text { leaf } \mathbf{j}^{\prime} \in T_{0} \backslash\{\mathbf{j}\} \text { is a descendant of }\left(j_{1}: j_{2}: \cdots: j_{q}\right)\right\} \subset \\
\subset \bigcup_{j \in \mathcal{J}_{i}} \bar{V}_{\left(j_{1}: j_{2}: \cdots: j_{q}: j\right)}=S_{j_{1}} \circ S_{j_{2}} \circ \cdots \circ S_{j_{q}}\left(F_{T_{i} \backslash\left\{\left(j_{q+1}\right)\right\}}\right), \quad \mathcal{J}_{i}=\left(j_{1}: j_{2}: \cdots: j_{q}: \cdot\right), \\
\bigcup\left\{A_{\mathbf{j}^{\prime}}^{\prime \mathbf{j}^{\prime}} \mid \text { leaf } \mathbf{j}^{\prime} \in T^{\prime} \text { is a descendant of }\left(j_{1}: j_{2}: \cdots: j_{q}\right)\right\}=S_{j_{1}} \circ S_{j_{2}} \circ \cdots \circ S_{j_{q}}\left(A^{\prime\left(j_{1}: j_{2}: \cdots: j_{q}\right)}\right),
\end{gathered}
$$

and the "common part" is outside of the open set $V_{j_{1}: j_{2}: \cdots: j_{q}}$.

This implies

$$
\begin{gathered}
h\left(A, F_{T_{0} \backslash\{\mathbf{j}\}}\right) \geqslant h\left(A^{\prime}, F_{T_{0} \backslash\{\mathbf{j}\}}\right) \geqslant \\
\geqslant h\left(S_{j_{1}} \circ S_{j_{2}} \circ \cdots \circ S_{j_{q}}\left(A^{\prime\left(j_{1}: j_{2}: \cdots: j_{q}\right)}\right), \partial V_{\left(j_{1}: j_{2}: \cdots: j_{q}\right)} \cup S_{j_{1}} \circ S_{j_{2}} \circ \cdots \circ S_{j_{q}}\left(F_{T_{i} \backslash\left\{\left(j_{q+1}\right)\right\}}\right)\right)= \\
=c_{j_{1}} c_{j_{2}} \ldots c_{j_{q}} h\left(A^{\prime\left(j_{1}: j_{2}: \cdots: j_{q}\right)}, \partial V \cup F_{T_{i} \backslash\left\{\left(j_{q+1}\right)\right\}}\right) \geqslant c_{j_{1}} c_{j_{2}} \ldots c_{j_{q}} \varepsilon_{i j_{q+1}}, \quad \mathcal{J}_{i}=\left(j_{1}: j_{2}: \cdots: j_{q}: \cdot\right) .
\end{gathered}
$$

Now the required inequality is at hand.

Remark 7. The number of all possible pairs $(i, j)$ such that $j \in \mathcal{J}_{i}$ is finite, hence $\varepsilon=$ $\min _{j \in \mathcal{J}_{i}} \varepsilon_{i j}>0$. Thus the inequality in the previous lemma can be simplified (and weakened): $\inf \left\{h\left(A, F_{T_{0} \backslash\{\mathbf{j}\}}\right) \mid A \in \mathcal{K}_{T}, T\right.$ is a finite $\mathbf{S}$-tree not having the vertex $\left.\mathbf{j}\right\} \geqslant c_{j_{1}} c_{j_{2}} \ldots c_{j_{r-1}} \varepsilon$.

We use also the following fact.

Lemma 3 ([3], Lemma 9.2). Let $\left\{V_{i}\right\}$ be a collection of disjoint open sets in $\mathbb{R}^{n}$ such that each $V_{i}$ contains a ball of radius $\alpha r$ and is contained in a ball of radius $\beta r$. Then any ball of radius $r$ intersects at most $\left(\frac{1+2 \beta}{\alpha}\right)^{n}$ of the closures $\bar{V}_{i}$.

Proof of the theorem. The support of $\mathcal{K}$ is contained in the attractor $K$ of IFS $\mathcal{S}$ for closed sets, for which we already know that $\operatorname{dim}_{H} K=\underline{\operatorname{dim}}_{B} K=\overline{\operatorname{dim}}_{B} K=s$. By monotonicity of dimensions

$\operatorname{dim}_{H} \operatorname{supp} \mathcal{K} \leqslant \operatorname{dim}_{H} K, \quad \underline{\operatorname{dim}}_{H} \operatorname{supp} \mathcal{K} \leqslant \underline{\operatorname{dim}}_{H} K, \quad \underline{\operatorname{dim}}_{B} \operatorname{supp} \mathcal{K} \overline{\operatorname{dim}}_{B} K$.

Taking into account $\underline{\operatorname{dim}}_{B} \mathcal{K} \leqslant \overline{\operatorname{dim}}_{B} \mathcal{K} \leqslant \overline{\operatorname{dim}}_{B} \operatorname{supp} \mathcal{K}$, we need only to prove $\underline{\operatorname{dim}}_{B} \mathcal{K} \geqslant s$.

Let $\mathcal{F}$ be a $\delta$-foundation of $\mathcal{K}$. Consider a finite subtree $T_{0}$ of the maximal $\mathcal{S}$-tree $T$ consisting of all vertices $\mathbf{j}=\left(j_{1}: j_{2}: \cdots: j_{r}\right)$ such that $c_{j_{1}} c_{j_{2}} \ldots c_{j_{r}} \varepsilon>\delta$ and their children. This implies that $c_{j_{1}} c_{j_{2}} \ldots c_{j_{r}} \varepsilon \leqslant \delta$ for all leaves of $T_{0}$. We prove that all sets $\bar{V}_{\mathbf{j}}$, $\mathbf{j}$ is a leaf of $T_{0}$, meet some element of $\mathcal{F}$. Assuming the contrary and having $\mathbf{j}$ fixed, it is easy to turn the $\mathcal{S}$-tree $T_{0}$ into an $\mathbf{S}$-tree $T_{0}^{\prime}$ containing $\mathbf{j}$ by dropping unnecessary branches. The set

$$
F_{T_{0}^{\prime}}=\left\{V_{\mathbf{j}^{\prime}} \mid \mathbf{j}^{\prime} \text { is a leaf of } T_{0}^{\prime}\right\}
$$


is in $\mathcal{K}$. There should be $D_{1}, D_{2}, \ldots, D_{l} \in \mathcal{F}$ such that each of them intersects some $V_{\mathbf{j}^{\prime}}$ for a leaf $\mathbf{j}^{\prime} \neq \mathbf{j}$, and $D_{1} \cup D_{2} \cup \cdots \cup D_{l} \in \mathcal{K}$. Then all minimal subsets $D \subset D_{1} \cup D_{2} \cup \cdots \cup D_{l}$ in $\mathcal{K}$ are determined with $\mathbf{S}$-trees not having a vertex $\mathbf{j}$, hence by Remark 7

$$
h\left(D_{1} \cup D_{2} \cup \cdots \cup D_{l}, F_{T_{0}^{\prime} \backslash\{\mathbf{j}\}}\right) \geqslant c_{j_{1}} c_{j_{2}} \ldots c_{j_{r-1}} \varepsilon>\delta,
$$

which contradicts to the assumption that all $D_{1}, D_{2}, \ldots, D_{l}$ have diameter $\leqslant \delta$ and intersect $F_{T_{0}^{\prime} \backslash\{\mathbf{j}\}}$. Therefore all $\bar{V}_{\mathbf{j}}$ for the leaves $\mathbf{j}$ of $T_{0}$ intersect some elements of $\mathcal{F}$.

The open set $V$ is bounded, hence contains a ball of radius $r_{1}$ and is contained in a ball of radius $r_{2}$. This implies that a set $V_{\left(j_{1}: j_{2}: \cdots: j_{r}\right)}$ contains a ball of radius

$$
c_{j_{1}} c_{j_{2}} \ldots c_{j_{r}} r_{1}=c_{j_{1}} c_{j_{2}} \ldots c_{j_{r-1}} \varepsilon \cdot \frac{r_{1}}{\varepsilon} c_{j_{r}}>\delta \frac{r_{1}}{\varepsilon} c_{\min }
$$

and is contained in a ball of radius

$$
c_{j_{1}} c_{j_{2}} \ldots c_{j_{r}} r_{2}=c_{j_{1}} c_{j_{2}} \ldots c_{j_{r}} \varepsilon \cdot \frac{r_{2}}{\varepsilon} \leqslant \delta \frac{r_{2}}{\varepsilon} .
$$

Each element of $\mathcal{F}$ has diameter $\leqslant \delta$, hence is contained in a ball of radius $\leqslant \delta$, and by Lemma 3 intersects at most

$$
M=\left(\frac{1+2 \frac{r_{2}}{\varepsilon}}{\frac{r_{1}}{\varepsilon} c_{\min }}\right)^{n}=\left(\frac{\varepsilon+2 r_{2}}{r_{1} c_{\min }}\right)^{n}
$$

of the sets $\bar{V}_{\mathbf{j}}$ for the leaves $\mathbf{j}$ of $T_{0}$. Now we estimate the number of these leaves.

It is straightforward to verify by induction that, for the solution $s$ of the equation $c_{1}^{s}+$ $c_{2}^{s}+\cdots+c_{k}^{s}=1$ and any finite $\mathcal{S}$-tree $T_{0}$, the sum

$$
\sum\left\{\left(c_{j_{1}} c_{j_{2}} \ldots c_{j_{r}}\right)^{s} \mid\left(j_{1}: j_{2}: \cdots: j_{r}\right) \text { is a leaf of } T_{0}\right\}
$$

is equal to 1 . Taking into account $c_{j_{1}} c_{j_{2}} \ldots c_{j_{r}} \varepsilon \leqslant \delta$, we obtain that all summands are not greater than $\left(\frac{\delta}{\varepsilon}\right)^{s}$, therefore the number of leaves is not less than $\left(\frac{\varepsilon}{\delta}\right)^{s}$. This implies that the minimal number $N_{\delta}(\mathcal{G})$ of elements in a $\delta$-foundation of $\mathcal{G}$ is not less than $\frac{1}{M}\left(\frac{\varepsilon}{\delta}\right)^{s}$. Now

$$
\underline{\operatorname{dim}}_{B} \mathcal{G}=\underline{\lim }_{\delta \rightarrow 0} \frac{\ln N_{\delta}(\mathcal{G})}{-\ln \delta} \geqslant \underline{\lim }_{\delta \rightarrow 0} \frac{\ln \left(\frac{1}{M}\left(\frac{\varepsilon}{\delta}\right)^{s}\right)}{-\ln \delta}=s,
$$

which completes the proof.

Remark 8. We cannot prove the similar equality for the Hausdorff dimension of a selfsimilar inclusion hyperspace using Mass Distribution Principle arguments like it was done in [3] for closed sets because a foundation needs not be a cover, hence the sum of the Hausdorff measures of the elements of a foundation cannot be estimated from below via the measure of the support. It is unknown yet whether the respective equality is valid.

If condition (iii) is not satisfied, we can give only an estimate for fractal dimensions of the attractor.

Theorem 2. Let $V \subset \mathbb{R}^{n}$ be a non-empty bounded open subset, $\mathbf{S}=\left\{\mathcal{S}_{1}, \mathcal{S}_{2}, \ldots, \mathcal{S}_{m}\right\}$ a finite family of finite sets of contracting similarities $\mathbb{R}^{n} \rightarrow \mathbb{R}^{n}$ such that:

(i) none of $\mathcal{S}_{i}$ is contained in another $\mathcal{S}_{j}$, i.e., each element of $\mathbf{S}$ is minimal w.r.t. inclusion; 
(ii) Open Set Condition holds for $\mathcal{S}=\bigcup \mathbf{S}=\left\{S_{1}, S_{2}, \ldots, S_{k}\right\}$ and $V$; and the subset of all $S \in \mathcal{S}$ that satisfy the condition

(iii*) for each element $A$ of the attractor $\mathcal{K}$ for $\mathbf{S}$ such that $A \subset \partial V$ and all $S^{\prime} \in \mathcal{S}$, $S^{\prime} \neq S$, we have $S^{\prime}(A) \not \subset S(\partial V)$

(we call such $S$ safe) is non-empty.

Then for the fixed point $\mathcal{K}$ of IFS $\Phi_{\mathbf{S}}$ for inclusion hyperspaces the inequality

$$
s_{0} \leqslant \underline{\operatorname{dim}}_{B} \mathcal{K} \leqslant \overline{\operatorname{dim}}_{B} \mathcal{K} \leqslant s=\operatorname{dim}_{H} \operatorname{supp} \mathcal{K}=\underline{\operatorname{dim}}_{B} \operatorname{supp} \mathcal{K}=\overline{\operatorname{dim}}_{B} \operatorname{supp} \mathcal{K}
$$

is valid, with $s$ and $s_{0}$ being the solutions of the equations $\sum_{1 \leqslant i \leqslant k} c_{i}^{s}=1, \sum_{\substack{1 \leqslant i \leqslant k \\ S_{i} \text { is safe }}} c_{i}^{s_{0}}=1$.

It is straightforward to modify appropriately the proof of the previous theorem. First, if $S_{j} \in \mathcal{S}_{i}$ is safe, then (iii*) implies $\varepsilon_{i j}>0$. Then we put $\varepsilon=\min \left\{\varepsilon \mid j \in \mathcal{J}_{i}, \varepsilon_{i j}>0\right\}$ and use Remark 7 only for the leaves $\mathbf{j}=\left(j_{1}: j_{2}: \cdots: j_{r}\right)$ of a finite $\mathbf{S}$-tree $T_{0}$ such that all $S_{j_{1}}$, $S_{j_{2}}, \ldots, S_{j_{r}}$ are safe. The sum of $\left(c_{j_{1}} c_{j_{2}} \ldots c_{j_{r}}\right)^{s_{0}}$ for such leaves is equal to 1 , which provides the required lower estimate.

Example 1. Consider the unit segment $I=[0,1]$ and the increasing affine bijections $S_{1}$ : $[0,1] \rightarrow[0,1 / 4], S_{2}:[0,1] \rightarrow[1 / 4,1 / 2], S_{3}:[0,1] \rightarrow[3 / 4,1]$. Then $\mathcal{S}=\left\{S_{1}, S_{2}, S_{3}\right\}$ and $V=$ $(0,1)$ satisfy Open Set Condition. For the IFS $\mathbf{S}=\left\{\left\{S_{1}, S_{2}\right\},\left\{S_{3}\right\}\right\}$ the minimal elements of the attractor $\mathcal{K}$ are obtained with the following procedure: of the unit segment we leave either two left quarters or the rightmost quarter, then we divide each of one or two quarters left into quarters as well and do with them the same, etc. The singleton $\{1\}$ is a unique element of $\mathcal{K}$ that is contained it the boundary of $V$ in $\mathbb{R}$. Its image $\{1 / 4\}$ under $S_{1}$ lies in the boundary $\{1 / 4,1 / 2\}$ of $S_{2}(V)$, hence $S_{2}$ is not safe $\left(S_{1}\right.$ and $S_{3}$ are). Therefore the lower and the upper box dimensions of $\mathcal{K}$ are between the solutions $s_{0}$ and $s$ of the equations $\left(\frac{1}{4}\right)^{s_{0}}+\left(\frac{1}{4}\right)^{s_{0}}=1$ and $\left(\frac{1}{4}\right)^{s}+\left(\frac{1}{4}\right)^{s}+\left(\frac{1}{4}\right)^{s}=1$, i.e., $1 / 2=s_{0} \leqslant \underline{\operatorname{dim}}_{B} \mathcal{K} \leqslant \overline{\operatorname{dim}}_{B} \mathcal{K} \leqslant s=\log _{4} 3$.

If $S_{1}$ was the decreasing affine bijection $[0,1] \rightarrow[0,1 / 4]$, with $S_{2}$ and $S_{3}$ the same, all $S_{i}$ would be safe, and we would have the precise value $\underline{\operatorname{dim}}_{B} \mathcal{K}=\overline{\operatorname{dim}}_{B} \mathcal{K}=s=\log _{4} 3$.

5. Dimensions for non-additive measures. The approach to capacities is similar to the one for inclusion hyperspaces: we try to approximate measures of all closed subsets of a metric compactum $X$ with measures of their approximations made of small "bricks" and count the necessary number of "bricks".

Definition 3. For a capacity $c \in \underline{M} X$ and a number $\varepsilon \geqslant 0$, a collection $\mathcal{F}$ of closed sets in $X$ is called an $\varepsilon$-foundation of $c$ if, for each non-empty $A \subset X$, there are $F_{1}, F_{2}, \ldots, F_{n} \in \mathcal{F}$, $n \in \mathbb{N}$, such that $F_{i} \cap A \neq \varnothing$, for all $1 \leqslant i \leqslant n$, and $c\left(F_{1} \cup F_{2} \cup \cdots \cup F_{n}\right) \geqslant c(A)-\varepsilon$.

Definition 4. An $\varepsilon$-foundation $\mathcal{F}$ of $c$ is called a $\delta$ - $\varepsilon$-foundation, for $\varepsilon \geqslant 0, \delta>0$, if $\operatorname{diam} F \leqslant \delta$ for all $F \in \mathcal{F}$.

Having a finite $\delta$ - $\varepsilon$-foundation $\mathcal{F}$ of a capacity $c$, we can define a capacity $c_{\mathcal{F}}$ as follows:

$$
c_{\mathcal{F}}(A)=c(\bigcup\{F \in \mathcal{F} \mid A \cap F \neq \varnothing\}), \quad A \underset{\mathrm{cl}}{\subset} X .
$$

It is easy to see that $\hat{d}\left(c, c_{\mathcal{F}}\right) \leqslant \max \{\delta, \varepsilon\}$, hence we obtain an approximation of a capacity $c$ with a capacity of much simpler nature, which is determined with a list of $2^{|\mathcal{F}|}$ values of $c$ for all finite unions of elements of $\mathcal{F}$. 
For $c \in \underline{M} X, s \geqslant 0, \varepsilon \geqslant 0, \delta>0$, we denote:

$$
\begin{gathered}
N_{\delta, \varepsilon}(c)=\min \{|\mathcal{F}| \mid \mathcal{F} \text { is a } \delta \text { - } \varepsilon \text {-foundation of } c\}, \\
\mathcal{H}_{\delta, \varepsilon}^{s}(c)=\inf \left\{\sum\left\{(\operatorname{diam} F)^{s} \mid F \in \mathcal{F}\right\} \mid \mathcal{F} \text { is a } \delta \text { - } \varepsilon \text {-foundation of } c\right\} .
\end{gathered}
$$

Observe that both values $N_{\delta, \varepsilon}(c)$ and $\mathcal{H}_{\delta, \varepsilon}^{s}(c)$ increase as any of $\delta$ and $\varepsilon$ decreases. Thus we can put $\mathcal{H}_{\varepsilon}^{s}(c)=\lim _{\delta \rightarrow 0} \mathcal{H}_{\delta, \varepsilon}^{s}(c)$ and define the weak upper box dimension, the weak lower box dimension, and the weak Hausdorff dimension of $c$ as follows:

$$
\begin{gathered}
\overline{\operatorname{dim}}_{\varepsilon B} c=\varlimsup_{\delta \rightarrow 0} \frac{\ln N_{\delta, \varepsilon}(c)}{-\ln \delta}, \quad \underline{\operatorname{dim}}_{\varepsilon B} c=\frac{\lim }{\delta \rightarrow 0} \frac{\ln N_{\delta, \varepsilon}(c)}{-\ln \delta}, \\
\operatorname{dim}_{\varepsilon H} c=\sup \left\{s \geqslant 0 \mid \mathcal{H}_{\varepsilon}^{s}(c)=\infty\right\}=\inf \left\{s \geqslant 0 \mid \mathcal{H}_{\varepsilon}^{s}(c)=0\right\}, \\
\overline{\operatorname{dim}}_{W B} c=\lim _{\varepsilon \rightarrow 0} \overline{\operatorname{dim}}_{\varepsilon B} c, \quad \underline{\operatorname{dim}}_{W B} c=\lim _{\varepsilon \rightarrow 0} \underline{\operatorname{dim}}_{\varepsilon B} c, \quad \operatorname{dim}_{W H} c=\lim _{\varepsilon \rightarrow 0} \operatorname{dim}_{\varepsilon H} c .
\end{gathered}
$$

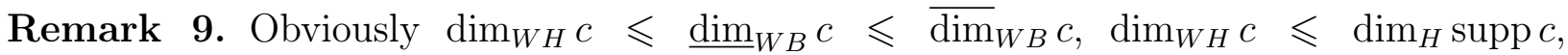
$\underline{\operatorname{dim}}_{W B} c \leqslant \underline{\operatorname{dim}}_{B} \operatorname{supp} c, \overline{\operatorname{dim}}_{W B} c \leqslant \overline{\operatorname{dim}}_{B} \operatorname{supp} c$ for all $c \in \underline{M} X$.

Proposition 4. Let a normalized capacity $c_{\mathcal{G}}$ be defined by an inclusion hyperspace $\mathcal{G}$ on a metric compactum $X$. Then the weak upper box dimension, the weak lower box dimension, and the weak Hausdorff dimension of $c_{\mathcal{G}}$ coincide respectively with the upper box dimension, the lower box dimension, and the Hausdorff dimension of $\mathcal{G}$.

Proof. It is sufficient to note that, for all $\varepsilon \in[0 ; 1)$ and $\delta>0, \mathcal{F}$ is a $\delta$ - $\varepsilon$-foundation for $c_{\mathcal{G}}$ if and only if it is a $\delta$-foundation for $\mathcal{G}$, therefore the considered values does not depend on $\varepsilon \in(0 ; 1)$.

The latter statement together with Proposition 2 implies an analogue of Example 7.1 in $[8]$ :

Proposition 5. There is a normalized capacity $c$ on $I^{2}$ such that $\underline{\operatorname{dim}}_{W B} c=\overline{\operatorname{dim}}_{W B} c<$ $\underline{\operatorname{dim}}_{B} \operatorname{supp} c=\overline{\operatorname{dim}}_{B} \operatorname{supp} c$.

Each additive regular measure on a metric compactum is a capacity, hence we apply the introduced dimensions to additive regular measures and compare them with the classical upper box dimension, lower box dimension, and Hausdorff dimension of a measure [8].

Observe that a collection $\mathcal{F}$ of closed subsets of $X$ is a $\delta$ - $\varepsilon$-foundation for a subprobability measure $\mu$ if and only if there are $F_{1}, F_{2}, \ldots, F_{n} \in \mathcal{F}$ such that diam $F_{i} \leqslant \delta$ for all $1 \leqslant i \leqslant n$ and $\mu\left(F_{1} \cup F_{2} \cup \cdots \cup F_{n}\right) \geqslant \mu(X)-\varepsilon$. In other words, $N_{\delta, \varepsilon}(c)$ is the minimal $n \in \mathbb{N}$ such that there is a set $F \underset{\text { cl }}{\subset} X$ that can be covered with $n$ sets of diameter $\leqslant \delta$, and $\mu(X \backslash F) \leqslant \varepsilon$. Hence $N_{\delta, \varepsilon}(c)$ is the minimum of $N_{\delta}(F)$ for all $F \underset{\mathrm{cl}}{\subset} X$ such that $\mu(F) \geqslant \mu(X)-\varepsilon$. Therefore, for a fixed such $F$ :

$$
\overline{\operatorname{dim}}_{\varepsilon B} \mu=\varlimsup_{\delta \rightarrow 0} \frac{\ln N_{\delta, \varepsilon}(\mu)}{-\ln \delta} \leqslant \varlimsup_{\delta \rightarrow 0} \frac{\ln N_{\delta}(F)}{-\ln \delta}=\overline{\operatorname{dim}}_{B} F,
$$

which implies $\overline{\operatorname{dim}}_{\varepsilon B} \mu \leqslant \inf \left\{\overline{\operatorname{dim}}_{B} F \mid F \underset{\text { cl }}{\subset} X, \mu(F) \geqslant \mu(X)-\varepsilon\right\}$. Thus

$$
\overline{\operatorname{dim}}_{W B} \mu=\lim _{\varepsilon \rightarrow 0} \overline{\operatorname{dim}}_{\varepsilon B} \mu \leqslant \lim _{\varepsilon \rightarrow 0} \inf \left\{\overline{\operatorname{dim}}_{B} F \mid F \underset{\mathrm{cl}}{\subset} X, \mu(F) \geqslant \mu(X)-\varepsilon\right\} .
$$


Analogously

$$
\underline{\operatorname{dim}}_{W B} \mu=\lim _{\varepsilon \rightarrow 0} \underline{\operatorname{dim}}_{\varepsilon B} \mu \leqslant \lim _{\varepsilon \rightarrow 0} \inf \left\{\underline{\operatorname{dim}}_{B} F \mid F \underset{\mathrm{cl}}{\subset} X, \mu(F) \geqslant \mu(X)-\varepsilon\right\} .
$$

Observe also

$$
\begin{aligned}
& \operatorname{dim}_{W H} \mu=\lim _{\varepsilon \rightarrow 0} \operatorname{dim}_{\varepsilon H} \mu=\lim _{\varepsilon \rightarrow 0} \inf \left\{s \geqslant 0 \mid \mathcal{H}_{\varepsilon}^{s}(\mu)=0\right\}= \\
& =\liminf _{\varepsilon \rightarrow 0}\left\{s \geqslant 0 \mid \text { for all } \delta>0, \theta>0 \text { there are } n \in \mathbb{N}, F_{1}, F_{2}, \ldots, F_{n} \subset X\right. \\
& \quad \text { such that } \operatorname{diam} F_{i} \leqslant \delta, \mu\left(F_{1} \cup F_{2} \cup \cdots \cup F_{n} \geqslant \mu(X)-\varepsilon,\right. \\
& \left.\quad \text { and }\left(\operatorname{diam} F_{1}\right)^{s}+\left(\operatorname{diam} F_{2}\right)^{s}+\cdots+\left(\operatorname{diam} F_{n}\right)^{s}<\theta\right\} \leqslant \\
& \leqslant \lim _{\varepsilon \rightarrow 0} \inf _{F \subset X} \inf \left\{s \geqslant 0 \mid \text { for all } \delta>0, \theta>0 \text { there are } n \in \mathbb{N}, F_{1}, F_{2}, \ldots, F_{n} \subset X\right. \\
& \qquad(F) \geqslant \mu(X)-\varepsilon \\
& \quad \operatorname{such} \text { that } \operatorname{diam} F_{i} \leqslant \delta, F_{1} \cup F_{2} \cup \cdots \cup F_{n} \supset F, \\
& \left.\quad \operatorname{and}\left(\operatorname{diam} F_{1}\right)^{s}+\left(\operatorname{diam} F_{2}\right)^{s}+\cdots+\left(\operatorname{diam} F_{n}\right)^{s}<\theta\right\}= \\
& =\liminf _{\varepsilon \rightarrow 0}\left\{\operatorname{dim}_{H}(F) \mid F \underset{\mathrm{cl}}{\subset} X, \mu(F) \geqslant \mu(X)-\varepsilon\right\} .
\end{aligned}
$$

The three latter rightmost expressions are almost exactly the definitions of the upper box dimension $\overline{\operatorname{dim}}_{B} \mu$, the lower box dimension $\underline{\operatorname{dim}}_{B} \mu$ and the Hausdorff dimension $\operatorname{dim}_{H} \mu$ of an additive regular measure $\mu$ in [8]. The only distinction is that in the mentioned definitions all Borel sets $F$ are considered, but due to inner compact regularity of a Borel measure this has no influence on the result.

Thus we arrive at the following result.

Proposition 6. If a subnormalized capacity $c$ is additive (i.e., is a subprobability measure), then the weak upper box dimension, the weak lower box dimension, and the weak Hausdorff dimension of $c$ do not exceed respectively the upper box dimension, the lower box dimension, and the Hausdorff dimension of $c$.

For lower box dimension we can make the latter statement more exact.

Proposition 7. For an additive regular measure $\mu$ on a metric compactum $X$ the weak lower box dimension and the lower box dimension coincide.

Proof. Taking into account $\underline{\operatorname{dim}}_{W B} \mu \leqslant \underline{\operatorname{dim}}_{B} \mu$, only the inequality $\underline{\operatorname{dim}}_{B} \mu \leqslant \underline{\operatorname{dim}}_{W B} \mu$ is to be proved. Assume $\underline{\operatorname{dim}}_{W B} \mu<\alpha$, then for all $\varepsilon>0$ by the definition of $\underline{\operatorname{dim}}_{W B} \mu$ there are $F_{1} \subset X$ and $\delta_{1}>0, \mu\left(X \backslash F_{1}\right)<\varepsilon / 2$, such that $\frac{\ln N_{\delta_{1}}\left(F_{1}\right)}{-\ln \delta_{1}}<\alpha \Longleftrightarrow N_{\delta_{1}}\left(F_{1}\right)<\delta_{1}^{\alpha}$.

Analogously there are $F_{2} \underset{c l}{\subset} X$ and $\delta_{2}>2 \delta_{1}, \mu\left(X \backslash F_{2}\right)<\varepsilon / 4$, such that $N_{\delta_{2}}\left(F_{2}\right)<\delta_{2}^{\alpha}$, $\ldots, F_{k} \underset{\text { cl }}{\subset} X$ and $\delta_{k}>2 \delta_{k-1}, \mu\left(X \backslash F_{k}\right)<\varepsilon / 2^{k}$, such that $N_{\delta_{k}}\left(F_{k}\right)<\delta_{k}^{\alpha}$, etc. Then for the closed set $F=\bigcap_{k=1}^{\infty} F_{k}$ we have

$$
\mu(X \backslash F)=\mu\left(\bigcup_{k=1}^{\infty} F_{k}\right) \leqslant \sum_{k=1}^{\infty} \mu\left(F_{k}\right) \leqslant \frac{\varepsilon}{2}+\frac{\varepsilon}{4}+\frac{\varepsilon}{8}+\cdots=\varepsilon,
$$

and $N_{\delta_{k}}(F) \leqslant N_{\delta_{k}}\left(F_{k}\right)<\delta_{k}^{\alpha}$ for all $k=1,2, \ldots$ Thus

$$
\varliminf_{\delta \rightarrow 0} \frac{\ln N_{\delta, \varepsilon}(\mu)}{-\ln \delta} \leqslant \varliminf_{k \rightarrow \infty} \frac{\ln N_{\delta_{k}}(F)}{-\ln \delta_{k}} \leqslant \alpha
$$


for all $\varepsilon>0$, hence $\underline{\operatorname{dim}}_{W B} \mu<\alpha \Longrightarrow \underline{\operatorname{dim}}_{B} \mu \leqslant \alpha$. This implies $\underline{\operatorname{dim}}_{W B} \mu \leqslant \underline{\operatorname{dim}}_{B} \mu$, therefore $\underline{\operatorname{dim}}_{W B}=\underline{\operatorname{dim}}_{B} \mu$.

Now we present an example that the weak upper box dimension of an additive measure can be strictly less that its upper box dimension.

Proposition 8. There is a probability measure $\mu$ on $I$ such that

$$
\underline{\operatorname{dim}}_{W B} \mu=\overline{\operatorname{dim}}_{W B} \mu=\underline{\operatorname{dim}}_{B} \mu<\overline{\operatorname{dim}}_{B} \mu .
$$

Proof. The following operations $\varphi, \psi$, and $\theta$ can be applied to any segment on the real line. We divide a segment into 8 equal parts and leave only the 2 -nd, the 4 -th, the 6 -th, and the 8-th part (together with the ends) for $\varphi$, the 4 -st and the 8-th part for $\psi$, and the 4-th part only for $\theta$. If we have a disjoint union of segments of equal length, then after applying $\varphi, \psi$, or $\theta$ to all of them we obtain a disjoint union of segments of 8 times less length. The Lebesgue measure of the union is multiplied by $\frac{1}{2}$, by $\frac{1}{4}$, and by $\frac{1}{8}$ respectively.

We order the set $\mathcal{P}$ of all pairs $(i, j)$ such that $i \in\{1,2, \ldots\}$ and $1 \leqslant j \leqslant 2^{i}$ lexicographically, i.e., $(i, j) \leqslant\left(i^{\prime}, j^{\prime}\right)$ iff either $i<i^{\prime}$ or $i=i^{\prime}$ and $j \leqslant j^{\prime}$. Let $b: \mathcal{P} \rightarrow \mathbb{N}$ be the increasing bijection. Observe that if $b(i, j)=k$, then $2+2^{2}+\cdots+2^{i-1}=2^{i}-2<k \leqslant$ $2+2^{2}+\cdots+2^{i-1}+2^{i}=2^{i+1}-2$.

Now we take the unit segment $I$ and construct a decreasing sequence of its non-empty closed sets $F_{1} \supset G_{1} \supset F_{2} \supset G_{2} \supset F_{3} \supset \ldots$ All $F_{i}$ will be disjoint unions of $2^{2^{k}}$ segments of equal length $\frac{1}{8^{k}}$, with pairwise distances between them not less than $\frac{1}{8^{2^{k}}}$. In particular, the set $F_{1}$ is equal to $[0,1 / 8] \cup[1 / 4,3 / 8] \cup[1 / 2,5 / 8] \cup[3 / 4,7 / 8]$. For all $k \in \mathbb{N}$, to obtain $F_{k+1}$, we proceed as follows: take $i \in \mathbb{N}, 1 \leqslant j \leqslant 2^{i}$ such that $b(i, j)=k$, then divide all $2 \cdot 2^{2^{k}}$ segments constituting $F_{k}$ from left to right into $2^{i}$ groups of equal total length, and put $A_{k}$ to be the union of the elements of $j$-th group. Apply the operation $\psi$ to all segments of $F_{k}$ that are not contained in $A_{k}$, then apply it to all the segments obtained, etc, and repeat this $2^{k-1}$ times. To the segments that are contained in $A_{k}$, we similarly apply the operation $\varphi$ iteratively $2^{k-1}$ times. The resulting set is denoted $G_{k}$. Next to its segments that are not in $A_{k}$ we apply $\psi 2^{k-1}$ more times, but to the segments in $A_{k}$ the operation $\theta$ is applied $2^{k-1}$ times, so we obtain the set $F_{k+1}$. It is easy to see that each segment of $F_{k}$ has been divided either into $2^{2^{k-1}} \cdot 2^{2^{k-1}}=2^{2^{k-1}+2^{k-1}}=2^{2^{k}}$ or into $4^{2^{k-1}} \cdot 1^{2^{k-1}}=2^{2 \cdot 2^{k-1}}=2^{2^{k}}$ parts of $8^{2^{k}}$ times less length, hence now in $F_{k+1}$ there are $2^{2^{k}} \cdot 2^{2^{k}}=2^{2^{k+1}}$ segments of length $\frac{1}{8^{2^{k}}} \cdot \frac{1}{8^{2^{k}}}=\frac{1}{8^{2^{k+1}}}$ each indeed.

There is a unique regular probability measure $\mu$ on $I$ with the support $F_{\infty}=\bigcap_{k=1}^{\infty} F_{k}$ such that all $2^{2^{k}}$ segments of $F_{k}$ have equal measure $\frac{1}{2^{2^{k}}}$. Consider fractal dimensions of $\mu$.

To find the weak upper box dimension of $\mu$, recall that $2^{i} \geqslant \frac{k}{2}+1$. For all $\varepsilon>0$ we can choose $k_{0} \in \mathbb{N}$ such that $\frac{k_{0}}{2}+1 \geqslant \frac{1}{\varepsilon}$, then for all $k \geqslant k_{0}$ the measure of $A_{k}$ is not greater than $\frac{1}{2^{i}} \leqslant \frac{1}{k / 2+1} \leqslant \varepsilon$. Without loss of generality we can assume that

$$
\frac{1}{8^{2^{k}}} \geqslant \frac{1}{8^{l}}>\delta \geqslant \frac{1}{8^{l+1}} \geqslant \frac{1}{8^{2^{k+1}}}
$$

for some $k \geqslant k_{0}$. For $F=F_{\infty} \backslash A_{k}$ we have $\mu(F) \geqslant 1-\varepsilon$. The set $F$ is contained in $F_{k} \backslash A_{k}$ and can be covered with segments of length $\frac{1}{8^{l+1}} \leqslant \delta$ obtained after $l+1-2^{k}$ iterations of $\psi$ applied to $\frac{2^{2^{k}}\left(2^{i}-1\right)}{2^{i}}$ segments of $F_{k} \backslash A_{k}$. Thus $N_{\delta}^{\varepsilon}(\mu) \leqslant N_{\delta}(F) \leqslant 2^{l+1-2^{k}} \cdot \frac{2^{2^{k}}\left(2^{i}-1\right)}{2^{i}} \leqslant 2^{l+1}<\frac{2}{\sqrt[3]{\delta}}$, therefore

$$
\varlimsup_{\varepsilon B} \mu \leqslant \varlimsup_{\delta \rightarrow 0} \frac{\ln (2 / \sqrt[3]{\delta})}{-\ln \delta}=\frac{1}{3},
$$


hence $\overline{\operatorname{dim}}_{W B} \mu \leqslant 1 / 3$.

On the other hand, for all $F \underset{\mathrm{cl}}{\subset} F_{\infty}$ such that $\mu(F) \geqslant 1-\varepsilon$ we have $\mu\left(F \backslash A_{k}\right) \geqslant 1-2 \varepsilon$, hence, of the segments of length $\frac{1}{8^{l}}>\delta$ and measure $\frac{1}{2^{l}}$ obtained after $l-2^{k}$ iterations of $\psi$ applied to the segments of $F_{k} \backslash A_{k}, F$ meets at least $(1-2 \varepsilon) 2^{l} \geqslant \frac{(1-2 \varepsilon) 2^{l}}{2 \sqrt[3]{\delta}}$. Gaps between these segments are also not less than $\frac{1}{8^{l}}>\delta$, hence each $\delta$-cover of $F \backslash A_{k}$ has $\geqslant \frac{1-2 \varepsilon}{2 \sqrt[3]{\delta}}$ elements. Thus

$$
\varliminf_{\varepsilon B}(\mu) \geqslant \varliminf_{\delta \rightarrow 0} \frac{\ln (1 / 2 \sqrt[3]{\delta})}{-\ln \delta}=\frac{1}{3}
$$

which yields $\underline{\operatorname{dim}}_{W B} \mu \geqslant 1 / 3 \Longrightarrow \underline{\operatorname{dim}}_{B} \mu=\underline{\operatorname{dim}}_{W B} \mu=\overline{\operatorname{dim}}_{W B} \mu=1 / 3$.

To estimate the upper box dimension of $\mu$, fix $\varepsilon>0$ and for each $i \in \mathbb{N}$ consider all $k \in \mathbb{N}$ such that $k=b(i, j)$ for some $j$, i.e., $2^{i}-1 \leqslant k \leqslant 2^{i+1}-2$. The family of $2^{i}$ sets $A_{2^{i}-1}, A_{2^{i}}$, $\ldots, A_{2^{i+1}-3}, A_{2^{i+1}-2}$ of measure $1 / 2^{i}$ each is a disjoint cover of $F_{\infty}$. Assume that $F \subset I$, $\mu(F) \geqslant 1-\varepsilon$ for $\varepsilon>0$. Then at least one of the intersections $F \cap A_{k}$ for $2^{i}-1 \leqslant k \leqslant 2^{i+1}-2$ has the measure $\geqslant \frac{1-\varepsilon}{2^{i}}$. Denote $k_{i}$ the respective index and consider the set $G_{k_{i}}$. It consists of segments of length $\frac{1}{8^{2^{k_{i}}}} \cdot \frac{1}{8^{2^{k_{i}-1}}}=\frac{1}{8^{3 \cdot 2^{k_{i}-1}}}$ with gaps between them not less than this length. There are $\frac{2^{2^{k_{i}}}}{2^{i}} \cdot 4^{2^{k_{i}-1}}=\frac{2^{2^{k_{i}+1}}}{2^{i}}$ of them in $G_{k_{i}} \cap A_{k_{i}}$, with the total measure $1 / 2^{i}$. Therefore $F$ meets al least $\frac{(1-\varepsilon) 2^{2^{k_{i}+1}}}{2^{i}}$ of these segments. Hence for $\delta_{i}=\frac{1}{8^{3 \cdot 2^{k_{i}-1}}}$ each $\delta_{i^{-}}$-cover of $F \cap A_{k_{i}}$ has $\geqslant \frac{(1-\varepsilon) 2^{k^{k_{i}+1}}}{2^{i}}$ elements. We obtain a lower estimate

$$
\overline{\operatorname{dim}}_{B} F \geqslant \varlimsup_{i \rightarrow \infty} \frac{\ln N_{\delta_{i}}(F)}{-\ln \delta_{i}} \geqslant \varlimsup_{i \rightarrow \infty} \frac{\ln \frac{(1-\varepsilon) 2^{2^{k_{i}+1}}}{2^{i}}}{\ln \left(8^{3 \cdot 2^{k_{i}-1}}\right)}=\varlimsup_{i \rightarrow \infty} \frac{2^{k_{i}+1}-i}{9 \cdot 2^{k_{i}-1}}=\frac{4}{9},
$$

which implies $\overline{\operatorname{dim}}_{B} \mu \geqslant 4 / 9$, hence

$$
\overline{\operatorname{dim}}_{B} \mu \neq \overline{\operatorname{dim}}_{W B} \mu=1 / 3 .
$$

Question 2. Is there an example that the strict inequality $\operatorname{dim}_{W H} c<\operatorname{dim}_{H} c$ can also occur?

Proposition 9. Let $\mathcal{F}$ be an $\varepsilon$-foundation of $c \in M X$ and $\mathcal{F}^{\prime}$ a collection of closed sets in $X$ such that each $F \in \mathcal{F}$ is contained in $\operatorname{Int} F^{\prime}$ for some $F^{\prime} \in \mathcal{F}^{\prime}$. Then $\mathcal{F}^{\prime}$ is an $\varepsilon^{\prime}$-foundation of the conjugate capacity $\tilde{c}$ for all $\varepsilon^{\prime}>\varepsilon$.

Proof. Let $\tilde{c}(T)=\alpha \in I$. Then $c(A) \leqslant 1-\alpha$ for all $A \underset{\mathrm{cl}}{\subset} X, A \cap T=\varnothing$. Consider the collection $\mathcal{A}=\left\{A \underset{\mathrm{cl}}{\subset} X \mid c(A) \geqslant 1-\alpha+\varepsilon^{\prime}\right\}$, which is closed in $\exp X$ w.r.t. the Vietoris topology. For each $A \in \mathcal{A}$ there are $F_{1}, F_{2}, \ldots, F_{n} \in \mathcal{F}$ such that $F_{i} \cap A \neq \varnothing$ for all $1 \leqslant i \leqslant n$, and

$$
c\left(F_{1} \cup F_{2} \cup \cdots \cup F_{n}\right) \geqslant c(A)-\varepsilon>1-\alpha,
$$

hence there is $F_{i} \in \mathcal{F}$ such that $F_{i} \cap T \neq \varnothing$. We denote it by $F_{A}$, and let $F_{A}^{\prime}$ be an element of $\mathcal{F}^{\prime}$ such that $F_{A} \subset \operatorname{Int} F_{A}^{\prime}$. Then the compactum $\mathcal{A}$ is covered with the open sets $\left\langle X, \operatorname{Int} F_{A}^{\prime}\right\rangle$, hence there is a finite subcover

$$
\left\langle X, \operatorname{Int} F_{A_{1}}^{\prime}\right\rangle \cup\left\langle X, \operatorname{Int} F_{A_{2}}^{\prime}\right\rangle \cup \cdots \cup\left\langle X, \operatorname{Int} F_{A_{k}}^{\prime}\right\rangle \supset \mathcal{A} .
$$


Now consider the set $F_{A_{1}}^{\prime} \cup F_{A_{2}}^{\prime} \cup \cdots \cup F_{A_{k}}^{\prime}$. If it has an empty intersection with $B \subset X$, then $B \notin \mathcal{A}$, hence $c(B)<1-\alpha+\varepsilon^{\prime}$. This implies $\tilde{c}\left(F_{A_{1}}^{\prime} \cup F_{A_{2}}^{\prime} \cup \cdots \cup F_{A_{k}}^{\prime}\right) \geqslant \alpha-\varepsilon^{\prime}$. Taking into account $F_{A_{j}}^{\prime} \in \mathcal{F}^{\prime}$ and $F_{A_{j}}^{\prime} \cap T \neq \varnothing$ for all $1 \leqslant j \leqslant k$, we obtain that $\mathcal{F}^{\prime}$ is an $\varepsilon^{\prime}$-foundation of the conjugate capacity $\tilde{c}$.

Corollary 2. The conjugacy mapping $\varkappa X: M X \rightarrow M X$ preserves the weak lower box dimension, the weak upper box dimension and the weak Hausdorff dimension of the normalized capacities.

6. Self-similar non-additive measures. As inclusion hyperspaces are tightly connected with capacities ( [12]), it is natural to go forth and define IFS for normalized capacities. As $M X$ for a metric compactum $X$ is a Lawson lattice w.r.t. "setwise" infs and sups, we can put $M \bar{r}(c)=\bigwedge_{r \in \bar{r}} M r(c)$ for all $\bar{r} \in \exp R_{q}(X)$ and $c \in M X$. Then $M \bar{r}(c)$ is in $M X$ and depends continuously on $(\bar{r}, c) \in \exp R_{q}(X) \times M X$. Now for $\mathcal{R} \in M R_{q}(X)$ the capacity $M \mathcal{R}(c)$ was defined in [7] with the formula

$$
M \mathcal{R}(c)(F)=\bigvee_{\bar{r} \in \exp R_{q}(X)} \min \{M \bar{r}(c)(F), \mathcal{R}(\bar{r})\} \text { for } F \underset{\mathrm{cl}}{\subset} X .
$$

This means that $M \mathcal{R}(c)(F) \geqslant a \in[0 ; 1]$ if and only if there is a non-empty closed set $\bar{r}$ of contractions with factors $\leqslant q$ such that $\mathcal{R}(\bar{r}) \geqslant a$ and $c\left(r^{-1}(F)\right) \geqslant a$ for all $r \in \bar{r}$. Theorem 2 [7] states that $M \mathcal{R}(c)$ is a capacity on $X$, and the mapping $M \mathcal{R}$ is non-expanding but is not a contraction. Nevertheless, there is a unique $c_{0} \in M X$ such that $M \mathcal{R}\left(c_{0}\right)=c_{0}$ (an attractor for the iterated function system $\mathcal{R}$ ), and for any $c \in M X$ we have $\hat{d}\left((M \mathcal{R})^{n}(c), c_{0}\right) \leqslant q^{n} \operatorname{diam} X$, thus $(M \mathcal{R})^{n}(c) \rightarrow c_{0}$ as $n \rightarrow \infty$.

We also simplify the above definition to consider capacities $\mathcal{R}$ with finite supports only. Assume that $\mathcal{R}$ is a capacity with the support $\mathcal{S}=\left\{S_{1}, S_{2}, \ldots, S_{k}\right\}, S_{j}$ are contracting similarities $\mathbb{R}^{n} \rightarrow \mathbb{R}^{n}$ that send a non-empty compact set $D$ into itself. Then $\mathcal{R}$ attains a finite number of values $0=a_{0}<a_{1}<a_{2}<\cdots<a_{v}=1$. It is uniquely determined with the sequence of its sections at the levels $a_{u}, 1 \leqslant u \leqslant v$, i.e., of the inclusion hyperspaces

$$
\mathcal{R}_{u}=\left\{\mathcal{F} \underset{\mathrm{cl}}{\subset} R_{q}(D) \mid \mathcal{R}(\mathcal{F}) \geqslant a_{u}\right\}
$$

(the section $\mathcal{R}_{0}$ is always equal to $\exp R_{q}(D)$ ). By the assumption the family $\mathbf{S}_{u}$ of minimal elements of $\mathcal{R}_{u}$ consists only of subsets of $\mathcal{S}$.

Therefore we define simplified IFS for normalized capacities as a pair of two sequences: $0=a_{0}<a_{1}<a_{2}<\cdots<a_{v}=1$ of numbers and $\mathbb{S}=\left(\mathbf{S}_{1}, \mathbf{S}_{2}, \ldots, \mathbf{S}_{v}\right)$ of non-empty families of non-empty subsets of $\mathcal{S}=\left\{S_{1}, S_{2}, \ldots, S_{k}\right\}$. We consider the sequence $\left(a_{u}\right)$ fixed and omit it in the denotations. The sequence $\mathbb{S}$ must satisfy the requirement that each set $\mathcal{S}^{\prime} \in \mathbf{S}_{u}$ contains a set $\mathcal{S}^{\prime \prime} \in \mathbf{S}_{u-1}, 2 \leqslant u \leqslant v$. Then, for a capacity $c \in M D$ and a set $A \underset{\text { cl }}{\subset} D$, the value $\Phi_{\mathbb{S}}(c)(A)$ is the largest $a \in(0 ; 1]$ such that there is $u \in\{1,2, \ldots, v\}, a \leqslant a_{0}$, and $\mathcal{S}^{\prime} \in \mathbf{S}_{u}$ such that for all $S \in \mathcal{S}^{\prime}$ the inequality $M S(c)(A) \geqslant a$ (i.e., $c\left(S^{-1}(A)\right) \geqslant a$ ) is valid. We assume $\max \varnothing=0$, i.e., if there is no such $a$, then $\Phi_{\mathbb{S}}(c)(A)=0$.

Proposition 10. Under the above assumptions the fixed point $c_{0}$ of $\Phi_{\mathbb{S}}$ attains only values $0=a_{0}<a_{1}<a_{2}<\cdots<a_{v}=1$ as well, and its sections $c_{0 u}=\left\{A \underset{\mathrm{cl}}{\subset} D \mid c_{0}(A) \geqslant a_{u}\right\}$ are inclusion hyperspaces in $D$ that are fixed points for $\Phi_{\mathbf{S}_{u}}, 1 \leqslant u \leqslant v$. 
The proof is straightforward and left to the reader.

Thus a self-similar normalized capacity is obtained. The following observation reduces the problem of calculation of fractal dimensions for capacities with finite sets of values to calculation of fractal dimensions for their sections.

Proposition 11. Let a normalized capacity $c$ on a metric compactum $X$ attain a finite set of values $0=a_{0}<a_{1}<a_{2}<\cdots<a_{v}=1$. Then the Hausdorff dimension, the lower weak box dimension, and the weak upper box dimension of $c$ are the maxima of the respective dimensions of the sections of $c$ at the levels $a_{u}, 1 \leqslant u \leqslant v$.

Proof. Observe first that, for $\delta>0$ and $0<\varepsilon<\min \left\{a_{u}-a_{u-1} \mid 1 \leqslant u \leqslant v\right\}$, a family $\mathcal{F}$ is a $\delta$ - $\varepsilon$-foundation for $c$ if and only if it is a $\delta$-foundation for all sections $c_{u}=\{A \underset{\mathrm{cl}}{\subset}$ $\left.X \mid c_{0}(A) \geqslant a_{u}\right\}, 1 \leqslant u \leqslant v$. It is equivalent to $\mathcal{F}$ being the union of $\delta$-foundations $\mathcal{F}_{u}$ for the inclusion hyperspaces $c_{u}$. Now the required equalities are at hand.

Now it is obvious how to estimate the weak lower and upper box dimensions for a selfsimilar capacity: we just apply Theorem 2 "sectionwise" to all sections $\mathbf{S}_{u}$, and then take two maxima, namely of the obtained lower estimates and of the upper estimates.

Concluding remarks. The suggested fractal dimensions for the regular non-additive measures on metric compacta embrace classic dimensions for the sets (via inclusion hyperspaces) and are less than or equal to dimensions for the regular additive measures. In this concern the following questions and problems arise.

The main question (partially answered in this paper): when do the introduced weak dimensions coincide with the classical ones and when do the strict inequalities hold?

Are the introduced dimension functions continuous w.r.t. suitably chosen topologies, e.g., are they upper or lower semicontinuous? If not, what are the points of (semi-)continuity?

Are there reasonable analogues of fine multifractal analysis for the considered objects?

Finally, efficient methods for calculating weak dimensions are yet to be elaborated. We are going to develop these topics in our next publications.

\section{REFERENCES}

1. M. Barnsley, J. Hutchinson, Ö. Stenflo, V-variable fractals and superfractals, preprint, 2003, arXiv.org: math $/ 0312314$.

2. G. Choquet, Theory of capacity, Ann. l'Institute Fourier, 5 (1953-1954), 131-295.

3. K. Falconer, Fractal Geometry: Mathematical Foundations and Applications, John Wiley \& Sons, 2003.

4. J.E. Hutchinson, L. Rüshendorf, Random fractal measures via the contraction method, Indiana Univ. Math. J., 47 (1998), 471-487.

5. Lin Zhou, Integral representation of continuous comonotonically additive functionals, Trans. Amer. Math. Soc., 350 (1998), №5, 1811-1822.

6. P. Mattila, M. Morán, J.-M. Rey, Dimension of a measure, Studia Math., 142 (2000), №3, 219-233.

7. O.R. Nykyforchyn, Fractal capacities and iterated function systems, Mathematical Gerold of Shevchenko Scientific Society, 5 (2008), 259-273.

8. Ya.B. Pesin, Dimension Theory in Dynamical Systems: Contemporary Views and Applications, The Chicago University Press, Chicago and London, 1997.

9. A. Schief, Separation properties for self-similar sets, Proc. Amer. Math. Soc., 122 (1994), №1, 111-115. 
10. D. Schmeidler, Subjective probability and expected utility without additivity, Econometrica, 57 (1989), $571-587$.

11. E.R. Vrscay, From fractal image compression to fractal-based methods in mathematics, in Fractals in Multimedia, ed. by M.F. Barnsley, D. Saupe and E.R. Vrscay, New York, Springer-Verlag, 2002.

12. M.M. Zarichnyi, O.R. Nykyforchyn, Capacity functor in the category of compacta, Sbornik: Mathematics, 199 (2008), №2, 159-184.

13. M. Zarichnyi, A. Teleiko, Categorical Topology of Compact Hausdorff Spaces, VNTL Publ., Lviv, 1999.

Kasimir the Great University in Bydgoszcz

Institute of Mathematics

12 Weyssenhoff Sq., Bydgoszcz, Poland

inna.hlushak81@gmail.com

Vasyl' Stefanyk Precarpathian National University

Department of Mathematics and Computer Science

57 Shevchenka St., Ivano-Frankivsk, Ukraine

oleh.nyk@gmail.com 